\title{
Trees of cylinders and canonical splittings
}

\author{
VINCENT GUIRARDEL \\ GILBERT LEVITT
}

\begin{abstract}
Let $T$ be a tree with an action of a finitely generated group $G$. Given a suitable equivalence relation on the set of edge stabilizers of $T$ (such as commensurability, coelementarity in a relatively hyperbolic group, or commutation in a commutative transitive group), we define a tree of cylinders $T_{c}$. This tree only depends on the deformation space of $T$; in particular, it is invariant under automorphisms of $G$ if $T$ is a JSJ splitting. We thus obtain $\operatorname{Out}(G)$-invariant cyclic or abelian JSJ splittings. Furthermore, $T_{c}$ has very strong compatibility properties (two trees are compatible if they have a common refinement).
\end{abstract}

20E08; 20F65, 20F67, 20E06

\section{Introduction}

In group theory, a JSJ splitting of a group $G$ is a splitting of $G$ (as a graph of groups) in which one can read any splitting of $G$ and which is maximal for this property; see Sela [28], Rips and Sela [25], Dunwoody and Sageev [8], Fujiwara and Papasoglu [11], Bowditch [1], and the authors' papers $[12 ; 15 ; 17]$. One needs to place restrictions on allowed edge groups, for instance one defines an abelian JSJ splitting by restricting to splittings over abelian groups. One sometimes considers relative JSJ splittings, by restricting to splittings in which certain subgroups are required to be elliptic.

In general, JSJ splittings are not unique, and there is a whole space of JSJ splittings called the JSJ deformation space; see Forester [10] and the authors' paper [17]. A deformation space $[9 ; 14]$ is the set of all splittings whose elliptic subgroups are prescribed (one usually also adds constraints on edge groups). A typical example of a deformation space is Culler and Vogtmann's Outer Space [7]. Splittings in the same deformation space are related by a finite sequence of simple moves $[9 ; 14 ; 6]$.

JSJ deformation spaces being canonical, they are endowed with a natural action of $\operatorname{Out}(G)$. As they are contractible [5; 14], this usually gives homological information about $\operatorname{Out}(G)$ (see eg [7]). 
However, deformation spaces of splittings, including JSJ deformation spaces, may also have some bad behaviour. For instance the action of $\operatorname{Out}(G)$ may fail to be cocompact; the deformation space may even fail to be finite dimensional.

Much more satisfying is the case when one has a canonical splitting rather than just a canonical deformation space. Such a splitting is invariant under automorphisms; in other words, it is a fixed point for the action of $\operatorname{Out}(G)$ on the deformation space containing it. A typical example is the virtually cyclic JSJ splitting of a one-ended hyperbolic group $G$ constructed by Bowditch [1] from the topology of the boundary of $G$. Having such a splitting gives much more precise information about $\operatorname{Out}(G)$ (see Sela [28] and the second author's paper [20]).

The goal of this paper is to introduce a general construction producing a canonical splitting (called the tree of cylinders) from a deformation space $\mathcal{D}$. Rather than splittings (or graphs of groups), we think in terms of trees equipped with an action of $G$. We always assume that $G$ is finitely generated.

The construction starts with a class $\mathcal{E}$ of allowed edge stabilizers, endowed with an admissible equivalence relation (see Definition 3.1). The main examples are commensurability, coelementarity and commutation (see Examples A, B and C below, and Sections 3.1 to 3.7). All trees are assumed to have edge stabilizers in $\mathcal{E}$.

Given a tree $T \in \mathcal{D}$, the equivalence relation on edge stabilizers partitions edges of $T$ into cylinders. An essential feature of an admissible relation is that cylinders are connected (they are subtrees of $T$ ). By definition, the tree of cylinders of $T$ is the tree $T_{c}$ dual to the covering of $T$ by its cylinders (see Definition 4.3).

Theorem 1 The tree of cylinders $T_{c}$ depends only on the deformation space $\mathcal{D}$ containing $T$.

Moreover, the assignment $T \mapsto T_{c}$ is functorial: any equivariant map $T \rightarrow T^{\prime}$ induces a natural cellular map $T_{c} \rightarrow T_{c}^{\prime}$ (mapping an edge to an edge or a vertex).

We often say that $T_{c}$ is the tree of cylinders of the deformation space. It is $\operatorname{Out}(G)-$ invariant if $\mathcal{D}$ is.

Examples Consider the graph of groups pictured on the left of Figure 1 (edge groups are infinite cyclic and attached to the boundary of punctured tori). Its fundamental group $G$ is hyperbolic, and the splitting depicted is a cyclic JSJ splitting. Its tree of cylinders is the splitting pictured on the right. It belongs to the same deformation space, but it is $\operatorname{Out}(G)$-invariant; in particular, it has a symmetry of order 3 (it is the splitting constructed by Bowditch [1]). 

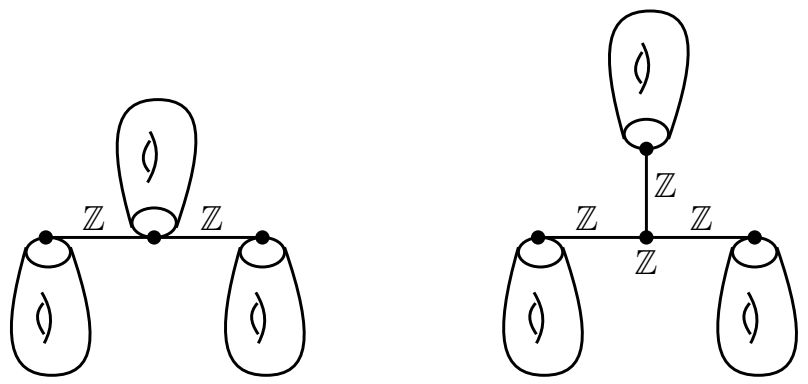

Figure 1: A JSJ splitting of a hyperbolic group and its tree of cylinders
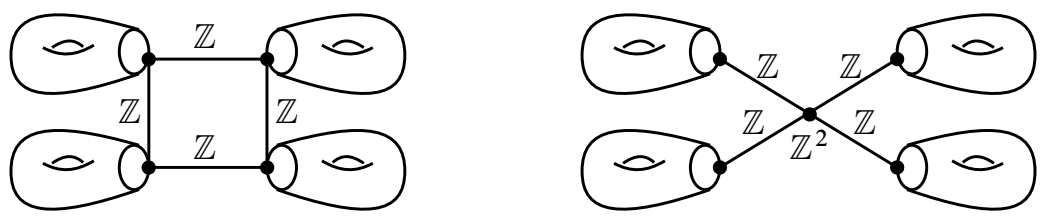

Figure 2: A JSJ splitting of a toral relatively hyperbolic group and its tree of cylinders

Now consider the graph of groups $\Gamma_{1}$ pictured on the left of Figure 2. Its fundamental group $G$ is a torsion-free toral relatively hyperbolic group. The splitting depicted is again a cyclic JSJ splitting, but no splitting in its deformation space $\mathcal{D}$ is $\operatorname{Out}(G)-$ invariant (there exist elements $t$ in the $\mathbb{Z}^{2}$ vertex group of the splitting $\Gamma_{2}$ pictured on the right such that twisting by $t$ around an edge of $\Gamma_{2}$ defines an automorphism having no fixed point in $\mathcal{D}$ ). The tree of cylinders of $\mathcal{D}$, which is $\operatorname{Out}(G)$-invariant, is the Bass-Serre tree of $\Gamma_{2}$. It does not belong to the same deformation space, because of the vertex with group $\mathbb{Z}^{2}$.

A basic property of $T_{c}$ is that it is dominated by $T$ : every subgroup which is elliptic (ie fixes a point) in $T$ is elliptic in $T_{c}$. But, as in the case of Culler and Vogtmann's Outer Space, it may happen that $T_{c}$ is trivial. For the construction to be useful, one has to be able to control how far $T_{c}$ is from $T$, the best situation being when $T_{c}$ and $T$ belong to the same deformation space (ie $T_{c}$ dominates $T$ ). One must also control edge stabilizers of $T_{c}$, as they may fail to be in $\mathcal{E}$.

Here are the main examples where this control is possible.

Example A $G$ is a hyperbolic group, $\mathcal{E}$ is the family of two-ended subgroups of $G$ and $\sim$ is the commensurability relation.

More generally, $G$ is hyperbolic relative to parabolic subgroups $H_{1}, \ldots, H_{n}$, the class $\mathcal{E}$ consists of the infinite elementary subgroups, and $\sim$ is the coelementarity 
relation ( $A \subset G$ is elementary if $A$ is virtually cyclic or is contained in a conjugate of some $H_{i}$, and $A \sim B$ if and only if $\langle A, B\rangle$ is elementary); one only considers trees in which each $H_{i}$ is elliptic.

Example B $G$ is torsion-free and CSA (centralizers of nontrivial elements are abelian and malnormal; for instance, the group considered in Figure 2 is CSA), $\mathcal{E}$ is the class of nontrivial abelian subgroups and $\sim$ is the commutation relation $(A \sim B$ if and only if $\langle A, B\rangle$ is abelian).

Example $\mathbf{C} G$ is torsion-free and commutative transitive (centralizers of nontrivial elements are abelian), $\mathcal{E}$ is the set of infinite cyclic subgroups of $G$ and $\sim$ is the commensurability relation.

Theorem 2 Let $G$ and $\mathcal{E}$ be as in Example A (resp. B). Let $T$ be a tree with edge stabilizers in $\mathcal{E}$, and assume that parabolic subgroups (resp. noncyclic abelian subgroups) are elliptic in $T$. Then

(1) $T_{c}$ has edge stabilizers in $\mathcal{E}$;

(2) $T_{c}$ belongs to the same deformation space as $T$;

(3) $T_{c}$ is almost 2-acylindrical.

A tree is $k$-acylindrical [27] if any segment $I$ of length $>k$ has trivial stabilizer. If $G$ has torsion, we use the notion of almost acylindricity: the stabilizer of $I$ is finite.

In general, edge stabilizers of $T_{c}$ may fail to be in $\mathcal{E}$. In this case, we also consider the collapsed tree of cylinders $T_{c}^{*}$, obtained from $T_{c}$ by collapsing all edges whose stabilizer is not in $\mathcal{E}$.

Theorem 3 Let $G$ and $\mathcal{E}$ be as in Example $C$. Let $T$ be a tree with infinite cyclic edge stabilizers, such that solvable Baumslag-Solitar subgroups of $G$ are elliptic in $T$. Then $T_{c}^{*}, T_{c}$ and $T$ lie in the same deformation space, and $T_{c}^{*}$ is 2-acylindrical.

Parabolic, abelian or Baumslag-Solitar subgroups as they appear in the hypotheses of Theorems 2 and 3 are always elliptic in $T_{c}$. If one does not assume that they are elliptic in $T$, they are the only way in which the deformation spaces of $T$ and $T_{c}^{*}$ differ (see Section 6 for precise statements).

In general, the deformation space of $T_{c}^{*}$ may be characterized by the following maximality property: $T_{c}^{*}$ dominates any tree $T^{\prime}$ such that $T$ dominates $T^{\prime}$ and cylinders 
of $T^{\prime}$ are bounded (Proposition 5.12). In many situations, one may replace boundedness of cylinders by acylindricity in the previous maximality statement (see Section 6).

Our results of Section 6 may then be interpreted as describing which subgroups must be made elliptic in order to make $T$ acylindrical. This is used in [18] to construct (under suitable hypotheses) JSJ splittings of finitely generated groups, using acylindrical accessibility.

Theorems 2 and 3 produce a canonical element in the deformation space of $T$. In particular, this provides canonical $\operatorname{Out}(G)$-invariant JSJ splittings.

Theorem 4 Let $G$ be hyperbolic relative to $H_{1}, \ldots, H_{n}$. Assume that $G$ is oneended relative to $H_{1}, \ldots, H_{n}$. There is an elementary (resp. virtually cyclic) JSJ tree relative to $H_{1}, \ldots, H_{n}$ which is invariant under the subgroup of $\operatorname{Out}(G)$ preserving the conjugacy classes of the $H_{i}$ 's.

The group $G$ is one-ended relative to $H_{1}, \ldots, H_{n}$ if there is no nontrivial tree with finite edge stabilizers in which each $H_{i}$ is elliptic.

When $G$ is a one-ended hyperbolic group, Theorem 4 yields the tree constructed by Bowditch [1].

Theorem 5 Let $G$ be a one-ended torsion-free CSA group. There exists an abelian (resp. cyclic) JSJ tree of $G$ relative to all noncyclic abelian subgroups, which is $\operatorname{Out}(G)-$ invariant.

In particular, one gets canonical cyclic and abelian JSJ splittings of toral relatively hyperbolic groups, including limit groups. See Bowditch [1], Bumagin, Kharlampovich and Miasnikov [2], Paulin [24] Scott and Swarup [26] and Papasoglu and Swenson [23] for other constructions of canonical JSJ splittings. As evidenced by the example on Figure 2, Theorems 4 and 5 do not hold for nonrelative JSJ splittings.

Scott and Swarup have constructed in [26] a canonical splitting over virtually polycyclic groups. We show in [16] that their splitting coincides (up to subdivision) with the tree of cylinders of the JSJ deformation space (with $\sim$ being commensurability; see Section 3.2).

In a forthcoming paper [19], we will use trees of cylinders to study some automorphism groups. In particular, we will use Theorem 4 to characterize relatively hyperbolic groups with infinite outer automorphism group (see also Carette [3] for the case of hyperbolic groups) and we will show that the splitting of Theorem 4 has the largest modular group. 
Another important feature of the tree of cylinders is compatibility. A tree $T$ is a refinement of $T^{\prime}$ if $T^{\prime}$ can be obtained from $T$ by equivariantly collapsing a set of edges. Two trees are compatible if they have a common refinement. Refinement is a notion much more rigid than domination: any two trees in Culler and Vogtmann's Outer Space dominate each other, but they are compatible if and only if they lie in a common simplex.

Theorem 6 Let $G$ be finitely presented and one-ended. Assume that $\mathcal{E}$ and $\sim$ are as in Examples $A, B$ or $C$.

If $T$ is a JSJ tree over subgroups of $\mathcal{E}$, then $T_{c}$ is compatible with every tree with edge stabilizers in $\mathcal{E}$ (in which parabolic subgroups are elliptic in Example $A$ ).

More general situations are investigated in Section 8. We show in [18] that under the hypotheses of Theorem 6 the tree $T_{c}^{*}$ is maximal (for domination) among trees which are compatible with every other tree. In other words, $T_{c}^{*}$ belongs to the same deformation space as the JSJ compatibility tree defined in [18].

The paper is organized as follows. After preliminaries, we define admissible equivalence relations and give examples (Section 3). In Section 4 we define the tree of cylinders. Besides the geometric definition sketched earlier, we give an algebraic one using elliptic subgroups and we show Theorem 1 (Corollary 4.10 and Proposition 4.11). In Section 5 we give basic properties of $T_{c}$. In particular, we show that most small subgroups of $G$ which are not virtually cyclic are elliptic in $T_{c}$. We also study boundedness of cylinders and acylindricity of $T_{c}^{*}$, we show $\left(T_{c}^{*}\right)_{c}^{*}=T_{c}^{*}$, and we prove the maximality property of $T_{c}^{*}$. Section 6 gives a description of the tree of cylinders in the main examples and proves Theorems 2 and 3 (they follow from Propositions 6.1, 6.3, 6.5). Section 7 recalls some material about JSJ splittings and proves the existence of canonical JSJ splittings as in Theorems 4 and 5. In Section 8 we study compatibility properties of the tree of cylinders and we prove Theorem 6 (it follows from Assertions (1) and (3) of Corollary 8.4 and Assertion (2) of Theorem 8.6).

\section{Preliminaries}

In this paper, $G$ will be a fixed finitely generated group.

Two subgroups $A$ and $B$ are commensurable if $A \cap B$ has finite index in both $A$ and $B$. We denote by $A^{g}$ the conjugate $g^{-1} A g$. The normalizer $N(A)$ of $A$ is the set of $g$ such that $A^{g}=A$. Its commensurator $\operatorname{Comm}(A)$ is the set of $g$ such that $g$ commensurates $A$ (ie $A^{g}$ is commensurable to $A$ ). The subgroup $A$ is malnormal if $A^{g} \cap A \neq\{1\}$ implies $g \in A$. 
If $\Gamma$ is a graph, we denote by $V(\Gamma)$ its set of vertices and by $E(\Gamma)$ its set of nonoriented edges.

A tree always means a simplicial tree $T$ on which $G$ acts without inversions. Given a family $\mathcal{E}$ of subgroups of $G$, an $\mathcal{E}$-tree is a tree whose edge stabilizers belong to $\mathcal{E}$. We denote by $G_{v}$ or $G_{e}$ the stabilizer of a vertex $v$ or an edge $e$.

A tree $T$ is nontrivial if there is no global fixed point, minimal if there is no proper $G$-invariant subtree. An element or a subgroup of $G$ is elliptic in $T$ if it has a global fixed point. An element which is not elliptic is hyperbolic. It has an axis on which it acts as a translation.

A subgroup $A$ consisting only of elliptic elements fixes a point if it is finitely generated, a point or an end in general. If a finitely generated subgroup $A$ is not elliptic, there is a unique minimal $A$-invariant subtree.

A group $A$ is slender if $A$ and all its subgroups are finitely generated. A slender group acting on a tree fixes a point or leaves a line invariant (setwise); see Dunwoody and Sageev [8].

A subgroup $A$ is small if it has no nonabelian free subgroups. As in [18], we could replace smallness by the following weaker property: whenever $G$ acts on a tree, $A$ fixes a point, or an end, or leaves a line invariant.

A tree $T$ dominates a tree $T^{\prime}$ if there is an equivariant map $f: T \rightarrow T^{\prime}$. Equivalently, any subgroup which is elliptic in $T$ is also elliptic in $T^{\prime}$. Having the same elliptic subgroups is an equivalence relation on the set of trees, the equivalence classes are called deformation spaces $[9 ; 14]$.

An equivariant map $f: T \rightarrow T^{\prime}$ between trees preserves alignment if $x \in[a, b] \Longrightarrow$ $f(x) \in[f(a), f(b)]$. Equivalently, $f$ is a collapse map: it is obtained by collapsing certain edges to points. In particular, $f$ does not fold.

We say that $T$ is a refinement of $T^{\prime}$ if there is a collapse map $f: T \rightarrow T^{\prime}$. Two trees $T$ and $T^{\prime}$ are compatible if they have a common refinement.

\section{Admissible relations}

Let $\mathcal{E}$ be a class of subgroups of $G$, stable under conjugation. It should not be stable under taking subgroups (all trees of cylinders are trivial if it is), but it usually is sandwich-closed: if $A \subset H \subset B$ with $A, B \in \mathcal{E}$, then $H \in \mathcal{E}$. Similarly, $\mathcal{E}$ is usually invariant under $\operatorname{Aut}(G)$. 
Definition 3.1 An equivalence relation $\sim$ on $\mathcal{E}$ is admissible if the following axioms hold for any $A, B \in \mathcal{E}$ :

(1) If $A \sim B$, and $g \in G$, then $A^{g} \sim B^{g}$.

(2) If $A \subset B$, then $A \sim B$.

(3) Let $T$ be an $\mathcal{E}$-tree. If $A \sim B$, and $A, B$ fix $a, b \in T$ respectively, then for each edge $e \subset[a, b]$ one has $G_{e} \sim A \sim B$.

More generally, one can require (3) to hold only for certain $\mathcal{E}$-trees. In particular, given subgroups $H_{i}$, we say that $\sim$ is admissible relative to the $H_{i}$ 's if (3) holds for all $\mathcal{E}$-trees $T$ in which each $H_{i}$ is elliptic.

Note that, given a subfamily $\mathcal{E}^{\prime} \subset \mathcal{E}$, stable under conjugation, the restriction of $\sim$ to $\mathcal{E}^{\prime}$ is admissible.

When proving that a relation is admissible, the only nontrivial part usually is axiom (3). The following criterion will be useful:

Lemma 3.2 If $\sim$ satisfies (1), (2) and axiom (3') below, then it is admissible.

(3') Let $T$ be an $\mathcal{E}$-tree. If $A \sim B$, and $A, B$ are elliptic in $T$, then $\langle A, B\rangle$ is also elliptic in $T$.

Proof We show axiom (3). Let $c$ be a vertex fixed by $\langle A, B\rangle$. Since $[a, b] \subset$ $[a, c] \cup[c, b]$, one may assume $e \subset[a, c]$. One has $A \subset G_{e}$ because $A$ fixes $[a, c]$, so that $G_{e} \sim A$ by axiom (2).

The lemma applies in a relative setting, with (3') restricted to trees in which each $H_{i}$ is elliptic.

Given an admissible relation $\sim$ on $\mathcal{E}$, we shall associate a tree of cylinders $T_{c}$ to any $\mathcal{E}$-tree $T$. If $\sim$ is only admissible relative to subgroups $H_{i}$, we require that each $H_{i}$ be elliptic in $T$.

Here are the main examples to which this will apply.

\subsection{Two-ended subgroups}

Let $\mathcal{E}$ be the set of two-ended subgroups of $G$ (a group $H$ is two-ended if and only if some finite index subgroup of $H$ is infinite cyclic). Commensurability, defined by $A \sim B$ if $A \cap B$ has finite index in both $A$ and $B$, is an admissible equivalence relation. 
Axiom (1) is clear. If $A, B$ are two-ended subgroups with $A \subset B$, then $A$ has finite index in $B$, so (2) holds. If $A, B \in \mathcal{E}$ are commensurable and fix points $a, b \in T$, then $A \cap B$ fixes $[a, b]$. If $e \subset[a, b]$, then $A \cap B \subset G_{e}$ (with finite index), and $G_{e} \sim A \cap B \sim A$.

Commensurability is admissible also on the set of infinite cyclic subgroups.

\subsection{Commensurability}

This class of examples generalizes the previous one. We used it in [16].

Let $\mathcal{E}$ be a conjugacy-invariant family of subgroups of $G$ such that

- any subgroup $A$ commensurable with some $B \in \mathcal{E}$ lies in $\mathcal{E}$;

- if $A, B \in \mathcal{E}$ are such that $A \subset B$, then $[B: A]<\infty$.

As in the previous example, one easily checks that commensurability is an admissible equivalence relation on $\mathcal{E}$.

For instance, $\mathcal{E}$ may consist of all subgroups of $G$ which are virtually $\mathbb{Z}^{n}$ for some fixed $n$, or all subgroups which are virtually polycyclic of Hirsch length exactly $n$. The classes of edge groups $\mathcal{Z K}$ considered by Dunwoody and Sageev in [8] also fit into this example.

\subsection{Coelementarity (splittings relative to parabolic groups)}

Let $G$ be hyperbolic relative to finitely generated subgroups $H_{1}, \ldots, H_{n}$. Recall that a subgroup of $G$ is parabolic if it is contained in a conjugate of an $H_{i}$, elementary if it is finite, or two-ended, or parabolic. If every $H_{i}$ is slender (resp. small), the elementary subgroups are the same as the slender (resp. small) subgroups.

We need the following standard fact (see for instance [22, Lemma 2.5, Theorem 4.3]).

Lemma 3.3 Any infinite elementary subgroup $H$ is contained in a unique maximal one, $E(H)$. This subgroup is two-ended or conjugate to an $H_{i}$.

Let $\mathcal{E}$ be the class of infinite elementary subgroups of $G$. Let $\sim$ be the coelementarity relation, defined by saying that $A, B \in \mathcal{E}$ are coelementary if and only if $\langle A, B\rangle$ is elementary.

Lemma 3.4 Coelementarity is an equivalence relation on $\mathcal{E}$.

Proof We have to prove transitivity. If $\langle A, B\rangle$ and $\langle B, C\rangle$ are elementary, since $B$ is infinite, $E(\langle A, B\rangle)=E(\langle B\rangle)=E(\langle B, C\rangle)$, so $\langle A, B, C\rangle \subset E(\langle B\rangle)$ is elementary. 
Coelementarity is admissible relative to the $H_{i}$ 's. Indeed, let us show axiom (3'), assuming that each $H_{i}$ is elliptic in $T$. The group $\langle A, B\rangle$ is elementary. It is clearly elliptic if it is parabolic. If it is two-ended, then it contains $A$ with finite index, so is elliptic in $T$ because $A$ is elliptic.

\subsection{Coelementarity (arbitrary splittings)}

We again assume $G$ is hyperbolic relative to finitely generated subgroups $H_{1}, \ldots, H_{n}$, and $\mathcal{E}$ is the family of infinite elementary subgroups. If we want to define a tree of cylinders for any $\mathcal{E}$-tree $T$, without assuming that the $H_{i}$ 's are elliptic in $T$, we need $\sim$ to be admissible (not just relative to the $H_{i}$ 's).

Lemma 3.5 Suppose that each $H_{i}$ is finitely-ended (ie $H_{i}$ is finite, virtually cyclic, or one-ended). Then coelementarity is admissible on $\mathcal{E}$.

Note that $H_{i}$ is finitely-ended if it is small (does not contain $F_{2}$ ).

Proof We show axiom (3), with $T$ any $\mathcal{E}$-tree ( $H_{i}$ is not required to be elliptic). Let $H=E(\langle A, B\rangle)$ be the maximal elementary subgroup containing both $A$ and $B$ (see Lemma 3.3). It is two-ended or conjugate to an infinite $H_{i}$.

If $H$ fixes a point $c \in T$, we argue as in the proof of Lemma 3.2. If $H$ is two-ended, it contains $A$ with finite index and therefore fixes a point in $T$. Thus, we can assume that $H$ is one-ended and does not fix a point in $T$. As $H$ is finitely generated, there is a minimal $H$-invariant subtree $T_{H} \subset T$.

The segment $[a, b]$ is contained in $\left[a, a^{\prime}\right] \cup\left[a^{\prime}, b^{\prime}\right] \cup\left[b^{\prime}, b\right]$ where $a^{\prime}$ (resp. $b^{\prime}$ ) is the projection of $a$ (resp. $b$ ) on $T_{H}$. If $e \subset\left[a, a^{\prime}\right]$, one has $A \subset G_{e}$ since $A$ fixes $\left[a, a^{\prime}\right]$, so that $A \sim G_{e}$. The same argument applies if $e \subset\left[b, b^{\prime}\right]$. Finally, assume $e \subset\left[a^{\prime}, b^{\prime}\right] \subset T_{H}$. Since $H$ is one-ended, the subgroup of $H$ stabilizing $e$ is infinite, so is in $\mathcal{E}$. Thus $G_{e} \sim\left(G_{e} \cap H\right) \sim H \sim A$. Axiom (3) follows.

\subsection{Commutation}

Recall that $G$ is commutative transitive if the commutation relation is a transitive relation on $G \backslash\{1\}$ (ie nontrivial elements have abelian centralizers). For example, torsion-free groups which are hyperbolic relative to abelian subgroups are commutative transitive.

Let $G$ be a commutative transitive group, and let $\mathcal{E}$ be the class of its nontrivial abelian subgroups. 
Lemma 3.6 The commutation relation, defined by $A \sim B$ if $\langle A, B\rangle$ is abelian, is admissible.

Proof It is an equivalence relation because of commutative transitivity. Axioms (1) and (2) are clear. We show axiom (3') (see Lemma 3.2). If $\langle A, B\rangle$ does not fix a point in $T$, the fixed point sets of $A$ and $B$ are disjoint, and $\langle A, B\rangle$ contains a hyperbolic element $g$. Being abelian, $\langle A, B\rangle$ then acts by translations on a line (the axis of $g$ ). But since $A$ and $B$ are elliptic, $\langle A, B\rangle$ fixes this line pointwise, a contradiction.

We will also consider a more restricted situation. Note that, in a commutative transitive group, any nontrivial abelian subgroup is contained in a unique maximal abelian subgroup, namely its centralizer.

Definition 3.7 $G$ is CSA if it is commutative transitive, and its maximal abelian subgroups are malnormal.

Since CSA is a closed property in the space of marked groups, $\Gamma$-limit groups for $\Gamma$ torsion-free hyperbolic are CSA (see Sela [29]); also see Champetier [4] for wilder examples.

\subsection{Finite groups}

Let $q \geq 1$ be an integer. Let $\mathcal{E}$ be the family of all subgroups of $G$ of cardinality $q$. It is easily checked that equality is an admissible equivalence relation on $\mathcal{E}$. This example will be used in [19].

\subsection{The equivalence relation of a deformation space}

Lemma 3.8 Let $\mathcal{E}$ be any conjugacy-invariant family of subgroups of $G$. The equivalence relation generated by inclusion is admissible relative to $\mathcal{E}$.

Proof We have to show axiom (3) holds, under the additional hypothesis that all groups of $\mathcal{E}$ are elliptic in $T$. Since $A \sim B$, we can find subgroups $A_{0}=A, A_{1}, \ldots, A_{n}=B$ in $\mathcal{E}$ where $A_{i}, A_{i+1}$ are nested (one contains the other). Let $u_{0}=a, u_{1}, \ldots, u_{n}=b$ be points of $T$ fixed by $A_{0}, \ldots, A_{n}$ respectively. Let $i$ be such that $e \subset\left[u_{i}, u_{i+1}\right]$, and assume for instance that $A_{i} \subset A_{i+1}$. Then $A_{i}$ fixes $e$, so $A_{i} \subset G_{e}$ and $G_{e} \sim A_{i} \sim A$.

In particular, let $\mathcal{D}$ be a deformation space (or a restricted deformation space in the sense of Definition 3.12 of [14]). The relation generated by inclusion is admissible on the family $\mathcal{E}$ consisting of generalized edge groups of reduced trees in $\mathcal{D}$ (see Section 4 of [14]). 


\section{The basic construction}

Let $\sim$ be an admissible equivalence relation on $\mathcal{E}$. We now associate a tree of cylinders $T_{c}$ to any $\mathcal{E}$-tree $T$. If $\sim$ is only admissible relative to subgroups $H_{i}$, we require that each $H_{i}$ be elliptic in $T$.

\subsection{Cylinders}

Definition 4.1 Let $T$ be an $\mathcal{E}$-tree (with each $H_{i}$ elliptic in $T$ in the relative case).

Define an equivalence relation $\sim_{T}$ on the set of (nonoriented) edges of $T$ by: $e \sim_{T} e^{\prime}$ if and only if $G_{e} \sim G_{e^{\prime}}$. A cylinder of $T$ is an equivalence class $Y$. We identify $Y$ with the union of its edges, a subforest of $T$.

A key feature of cylinders is their connectedness:

Lemma 4.2 Every cylinder is a subtree.

Proof Assume that $G_{e} \sim G_{e^{\prime}}$. By axiom (3), any edge $e^{\prime \prime}$ contained in the arc joining $e$ to $e^{\prime}$ satisfies $G_{e} \sim G_{e^{\prime}} \sim G_{e^{\prime \prime}}$, thus belongs to the same cylinder as $e$ and $e^{\prime}$.

Two distinct cylinders meet in at most one point. One can then define the tree of cylinders of $T$ as the tree $T_{c}$ dual to the covering of $T$ by its cylinders, as in [13, Definition 4.8]:

Definition 4.3 The tree of cylinders of $T$ is the bipartite tree $T_{c}$ with vertex set $V\left(T_{c}\right)=V_{0}\left(T_{c}\right) \sqcup V_{1}\left(T_{c}\right)$ defined as follows:

(1) $V_{0}\left(T_{c}\right)$ is the set of vertices $x$ of $T$ belonging to (at least) two distinct cylinders.

(2) $V_{1}\left(T_{c}\right)$ is the set of cylinders $Y$ of $T$.

(3) There is an edge $\varepsilon=(x, Y)$ between $x \in V_{0}\left(T_{c}\right)$ and $Y \in V_{1}\left(T_{c}\right)$ if and only if $x$ (viewed as a vertex of $T$ ) belongs to $Y$ (viewed as a subtree of $T$ ).

Alternatively, one can define the boundary $\partial Y$ of a cylinder $Y$ as the set of vertices of $Y$ belonging to another cylinder, and obtain $T_{c}$ from $T$ by replacing each cylinder by the cone on its boundary.

It is easy to see that $T_{c}$ is indeed a tree [13]. Here are a few other simple observations. If $\mathcal{E}^{\prime} \subset \mathcal{E}$, and $T$ is an $\mathcal{E}^{\prime}$-tree, its tree of cylinders as an $\mathcal{E}^{\prime}$-tree (with respect to the restricted admissible relation) is the same as $T_{c}$. 
The group $G$ acts on $T_{c}$ without inversions. It follows from [13, Lemma 4.9] that $T_{c}$ is minimal if $T$ is minimal. But $T_{c}$ may be a point, for instance if all edge stabilizers of $T$ are trivial.

Any vertex stabilizer $G_{v}$ of $T$ fixes a point in $T_{c}$ : the vertex $v$ of $V_{0}\left(T_{c}\right)$ if $v$ belongs to two cylinders, the vertex $Y$ of $V_{1}\left(T_{c}\right)$ if $Y$ is the only cylinder containing $v$. In other words, $T$ dominates $T_{c}$.

A vertex $x \in V_{0}\left(T_{c}\right)$ may be viewed either as a vertex of $T$ or as a vertex of $T_{c}$; its stabilizer in $T_{c}$ is the same as in $T$. The stabilizer of a vertex in $V_{1}\left(T_{c}\right)$ is the (global) stabilizer $G_{Y}$ of a cylinder $Y \subset T$; it may fail to be elliptic in $T$ (for instance if $T_{c}$ is a point and $T$ is not), so $T_{c}$ does not always dominate $T$. This will be studied in Sections 5 and 6.

Let us now consider edge stabilizers. We note:

Remark 4.4 Edge stabilizers of $T_{c}$ are elliptic in $T$, and they always contain a group in $\mathcal{E}$ : if $\varepsilon=(x, Y)$ and $e$ is an edge of $Y$ incident on $x$, then $G_{\varepsilon} \supset G_{e}$.

However, edge stabilizers of $T_{c}$ are not necessarily in $\mathcal{E}$. For this reason, it is convenient to introduce the collapsed tree of cylinders:

Definition 4.5 Given an $\mathcal{E}$-tree $T$, the collapsed tree of cylinders $T_{c}^{*}$ is the $\mathcal{E}$-tree obtained from $T_{c}$ by collapsing all edges whose stabilizer is not in $\mathcal{E}$.

\subsection{Algebraic interpretation}

We give a more algebraic definition of $T_{c}$, by viewing it as a subtree of a bipartite graph $Z$ defined algebraically using only information on $\mathcal{E}$, $\sim$, and elliptic subgroups of $T$. This will make it clear that $T_{c}$ only depends on the deformation space of $T$ (Corollary 4.10). We motivate the definition of $Z$ by a few observations.

If $Y \subset T$ is a cylinder, all its edges have equivalent stabilizers, and we can associate to $Y$ an equivalence class $\mathcal{C} \in \mathcal{E} / \sim$. We record the following for future reference.

Remark 4.6 Given an edge $\varepsilon=(x, Y)$ of $T_{c}$, let $e$ be an edge of $Y$ adjacent to $x$ in $T$. Then $G_{e}$ is a representative of the class $\mathcal{C}$ and is contained in $G_{\varepsilon}=G_{X} \cap G_{Y}$. If $G_{\mathcal{E}}$ belongs to $\mathcal{E}$, then it is in $\mathcal{C}$ by axiom (2) of admissible relations. In particular, if all edge stabilizers of $T_{c}$ are in $\mathcal{E}$, then $\left(T_{c}\right)_{c}=T_{c}$. Also note that $G_{Y}$ represents $\mathcal{C}$ if $G_{Y} \in \mathcal{E}$. 
Depending on the context, it may be convenient to think of a cylinder either as a set of edges of $T$, or a subtree $Y$ of $T$, or a vertex of $T_{c}$, or an equivalence class $\mathcal{C}$. Similarly, there are several ways to think of $x \in V_{0}\left(T_{c}\right)$ : as a vertex of $T$, a vertex of $T_{c}$, or an elliptic subgroup $G_{x}$.

If $x$ is a vertex of $T$ belonging to two cylinders, then its stabilizer $G_{x}$ is not contained in a group of $\mathcal{E}$ : otherwise all edges of $T$ incident to $x$ would have equivalent stabilizers by axiom (2), and $x$ would belong to only one cylinder.

More generally, let $v$ be any vertex of $T$ whose stabilizer is not contained in a group of $\mathcal{E}$. Then $G_{v}$ fixes $v$ only, and is a maximal elliptic subgroup. Conversely, let $H$ be a subgroup which is elliptic in $T$, is not contained in a group of $\mathcal{E}$, and is maximal for these properties. Then $H$ fixes a unique vertex $v$ and equals $G_{v}$.

Definition 4.7 Given an $\mathcal{E}$-tree $T$, let $Z$ be the bipartite graph with vertex set $V(Z)=V_{0}(Z) \sqcup V_{1}(Z)$ defined as follows:

(1) $V_{0}(Z)$ is the set of subgroups $H$ which are elliptic in $T$, not contained in a group of $\mathcal{E}$, and maximal for these properties.

(2) $V_{1}(Z)$ is the set of equivalence classes $\mathcal{C} \in \mathcal{E} / \sim$.

(3) There is an edge $\varepsilon$ between $H \in V_{0}(Z)$ and $\mathcal{C} \in V_{1}(Z)$ if and only if $H$ contains a group of $\mathcal{C}$.

As previously observed, $V_{0}(Z)$ may be viewed as the set of vertices of $T$ whose stabilizer is not contained in a group of $\mathcal{E}$.

It also follows from the previous observations that there is a natural embedding of bipartite graphs $j: T_{c} \rightarrow Z$ : for $v \in V_{0}\left(T_{c}\right)$, we define $j(v)=G_{v} \in V_{0}(Z)$; for $Y \in V_{1}\left(T_{c}\right)$, with associated equivalence class $\mathcal{C}$, we define $j(Y)=\mathcal{C} \in V_{1}(Z)$. Note that $j$ is well defined on $E\left(T_{c}\right)$ since adjacent vertices of $T_{c}$ have adjacent images in $Z$ by Remark 4.6.

A vertex $H \in V_{0}(Z)$ is a stabilizer $G_{v}$ for a unique $v \in T$. It is in $j\left(T_{c}\right)$ if and only if $v$ belongs to two cylinders. A vertex $\mathcal{C} \in V_{1}(Z)$ is in $j\left(T_{c}\right)$ if and only if some representative of $\mathcal{C}$ fixes an edge of $T$.

Proposition 4.8 Assume that the action of $G$ on $T$ is minimal and nontrivial, and that $T_{c}$ is not a point. Then $j\left(T_{c}\right)$ is the set of edges and vertices of $Z$ which are contained in the central edge of a segment of length 5 of $Z$.

Proof The action of $G$ on $T_{c}$ is minimal (see above) and nontrivial, so any edge of $T_{c}$ and of $j\left(T_{c}\right)$ is the central edge of a segment of length 5. The converse is an immediate consequence of items (1), (3), (4) of the following lemma. 
Lemma 4.9 (1) A vertex $v \in V_{1}(Z)$ belongs to $j\left(T_{c}\right)$ if and only if $v$ has valence at least 2 in $Z$.

(2) If $x \in T$ and $G_{x} \in V_{0}(Z)$ is adjacent to $j(Y) \in j\left(T_{c}\right)$, then $x \in Y$.

(3) An element of $V_{0}(Z)$ belongs to $j\left(T_{c}\right)$ if and only if it has at least 2 neighbours in $j\left(T_{c}\right)$.

(4) An edge of $Z$ lies in $j\left(E\left(T_{c}\right)\right)$ if and only if its endpoints are in $j\left(V\left(T_{c}\right)\right)$.

Proof In statements (1), (3) and (4), the direct implications are clear.

(1) Consider $\mathcal{C} \in V_{1}(Z)$ of valence at least 2. It has representatives $A \subset G_{X}$ and $B \subset G_{x^{\prime}}$ with $x, x^{\prime}$ distinct points of $T$. By axiom (3) of admissible relations, all edges in $\left[x, x^{\prime}\right]$ have stabilizer in $\mathcal{C}$. This shows $\mathcal{C} \in j\left(V_{1}\left(T_{c}\right)\right)$.

(2) Consider $G_{x} \in V_{0}(Z)$ adjacent to $\mathcal{C}=j(Y) \in V_{1}(Z)$ for some cylinder $Y$ of $T$. Then $\mathcal{C}$ has a representative $A \subset G_{x}$. Let $e$ be an edge of $Y$, so that $A \sim G_{e}$. By axiom (3), the cylinder $Y$ contains the arc joining $e$ to $x$, so $x \in Y$.

(3) If $G_{x} \in V_{0}(Z)$ is adjacent to $j(Y)$ and $j\left(Y^{\prime}\right)$, then $x \in Y \cap Y^{\prime}$ by statement (2), so $x \in V_{0}\left(T_{c}\right)$, and $G_{x} \in j\left(V_{0}\left(T_{c}\right)\right)$.

(4) Let $j(x)$ and $j(Y)$ be vertices of $j\left(T_{c}\right)$ joined by an edge of $Z$. Then $x \in Y$ by statement (2), so $x$ and $Y$ are joined by an edge in $T_{c}$.

Corollary 4.10 If $T, T^{\prime}$ are minimal nontrivial $\mathcal{E}$-trees belonging to the same deformation space, there is a canonical equivariant isomorphism between their trees of cylinders.

Proof The key observation is that the graph $Z$ is defined purely in terms of the elliptic subgroups, which are the same for $T$ and $T^{\prime}$. The corollary is trivial if $T_{c}, T_{c}^{\prime}$ are both points. If $T_{c}$ is not a point, then $V_{1}(Z)$ contains infinitely many vertices of valence $\geq 2$. As shown above (statement (1) of Lemma 4.9), this implies that $T_{c}^{\prime}$ is not a point, and the result follows directly from Proposition 4.8 .

\subsection{Functoriality}

There are at least two other ways of proving that $T_{c}$ only depends on the deformation space of $T$. One is based on the fact that any two trees in the same deformation space are connected by a finite sequence of elementary expansions and collapses [9]. One checks that these moves do not change $T_{c}$.

Another approach is to study the effect of an equivariant map $f: T \rightarrow T^{\prime}$ on the trees of cylinders. We always assume that $f$ maps a vertex to a vertex, and an edge to a point or an edge path. If $T^{\prime}$ is minimal, any $f$ is surjective. We say that $f$ is cellular if it maps an edge to an edge or a vertex. 
Proposition 4.11 Let $T, T^{\prime}$ be minimal $\mathcal{E}$-trees, and $f: T \rightarrow T^{\prime}$ an equivariant map. Let $T_{c}, T_{c}^{\prime}$ be the trees of cylinders of $T$ and $T^{\prime}$. Then $f$ induces a cellular equivariant map $f_{c}: T_{c} \rightarrow T_{c}^{\prime}$. This map does not depend on $f$, and is functorial in the sense that $(f \circ g)_{c}=f_{c} \circ g_{c}$.

Corollary 4.10 easily follows from this proposition. The proposition may be proved by considering the bipartite graph $Z$, but we give a geometric argument.

Proof We may assume that $T_{c}^{\prime}$ is not a point.

Lemma 4.12 Consider an equivariant map $f: T \rightarrow T^{\prime}$. For each cylinder $Y \subset T$, the image $f(Y)$ is either a cylinder $Y^{\prime}$ of $T^{\prime}$ or a point $p^{\prime} \in T^{\prime}$.

Proof If an edge $e^{\prime}$ of $T^{\prime}$ is contained in the image of an edge $e \subset Y$, then $G_{e^{\prime}}$ contains $G_{e}$, hence is equivalent to $G_{e}$ by axiom (2). This shows that $f(Y)$, if not a point, is contained in a unique cylinder $Y^{\prime}$. Conversely, if $e^{\prime}$ is an edge of $Y^{\prime}$, then any edge $e$ such that $e^{\prime} \subset f(e)$ satisfies $G_{e} \subset G_{e^{\prime}}$ hence is in $Y$. Thus $Y^{\prime}=f(Y)$.

We say that a cylinder $Y$ is collapsed if $f(Y)$ is a point $p^{\prime} \in T^{\prime}$. We claim that such a $p^{\prime}$ belongs to two distinct cylinders of $T^{\prime}$, so represents an element of $V_{0}\left(T_{c}^{\prime}\right)$. Consider the union of all collapsed cylinders, and the component containing $Y$. It is not the whole of $T$, so by minimality of $T$ it has at least two boundary points. These points belong to distinct noncollapsed cylinders whose images are the required cylinders containing $p^{\prime}$.

Also note that, if $x \in T$ belongs to two cylinders, so does $f(x)$. This is clear if $x$ belongs to no collapsed cylinder, and follows from the previous fact if it does.

This allows us to define $f_{c}$ on vertices of $T_{c}$, by sending $x \in V_{0}\left(T_{c}\right)$ to $f(x) \in V_{0}\left(T_{c}^{\prime}\right)$, and $Y \in V_{1}\left(T_{c}\right)$ to $Y^{\prime} \in V_{1}\left(T_{c}^{\prime}\right)$ or $p^{\prime} \in V_{0}\left(T_{c}^{\prime}\right)$. If $(x, Y)$ is an edge of $T_{c}$, then $f_{c}(x)$ and $f_{c}(Y)$ are equal or adjacent in $T_{c}^{\prime}$.

We may describe $f_{c}$ without referring to $f$, as follows. The image of $x \in V_{0}\left(T_{c}\right)$ is the unique point of $T^{\prime}$ fixed by $G_{x}$. The image of $Y$ is the unique cylinder whose edge stabilizers are equivalent to those of $Y$, or the unique point of $T^{\prime}$ fixed by stabilizers of edges of $Y$. Functoriality is easy to check.

Remark 4.13 Note that, if two edges $\left(x_{1}, Y_{1}\right)$ and $\left(x_{2}, Y_{2}\right)$ of $T_{c}$ are mapped by $f_{c}$ onto the same edge of $T_{c}^{\prime}$, then $Y_{1}=Y_{2}$. In particular, if the restriction of $f$ to each cylinder is either constant or injective, then $f_{c}$ preserves alignment. 


\section{General properties}

\subsection{The deformation space of $T_{c}$}

We fix $\mathcal{E}$ and an admissible relation $\sim$. We have seen that $T$ always dominates $T_{c}$ : any vertex stabilizer of $T$ fixes a point in $T_{c}$. Conversely, $T_{c}$ has two types of vertex stabilizers. If $v \in V_{0}\left(T_{c}\right)$, then its stabilizer is a vertex stabilizer of $T$, and $G_{v} \notin \mathcal{E}$ (see Section 4.2). On the other hand, the stabilizer $G_{Y}$ of a vertex $Y \in V_{1}\left(T_{c}\right)$ may fail to be elliptic in $T$. This means that $T_{c}$ is not necessarily in the same deformation space as $T$.

There are various ways to think of $G_{Y}$. It consists of those $g \in G$ that map the cylinder $Y$ to itself. If $e$ is any edge in $Y$, then $G_{Y}$ is the set of $g \in G$ such that $g G_{e} g^{-1} \sim G_{e}$. If $\mathcal{C}$ is the equivalence class associated to $Y$, then $G_{Y}$ is the stabilizer of $\mathcal{C}$ for the action of $G$ by conjugation on $\mathcal{E} / \sim$.

In Sections 3.1 and 3.2, the group $G_{Y}$ is the commensurator of $G_{e}$, for any $e \subset Y$. In Sections 3.3 and 3.4, it is the maximal elementary subgroup containing $G_{e}$. In Section 3.5, it is the normalizer of the maximal abelian subgroup $A$ containing $G_{e}$ (it equals $A$ if $G$ is CSA). In Section 3.6, it is the normalizer of $G_{e}$.

We first note:

Lemma 5.1 If $T$ is minimal, then $G_{Y}$ acts on $Y$ with finitely many orbits of edges.

Proof By minimality, there are finitely many $G$-orbits of edges in $T$. If two edges of $Y$ are in the same orbit under some $g \in G$, then $g$ preserves $Y$, so they are in the same orbit under $G_{Y}$.

Proposition 5.2 Given $T$, the following statements are equivalent:

(1) $T_{c}$ belongs to the same deformation space as $T$.

(2) Every stabilizer $G_{Y}$ is elliptic in $T$.

(3) Every cylinder $Y \subset T$ is bounded.

(4) No cylinder contains the axis of a hyperbolic element of $G$.

Proof We have seen (1) $\Leftrightarrow(2)$. If $Y$ is bounded, then $G_{Y}$ fixes a point of $T$ (the "center" of $Y$ ). If $G_{Y}$ fixes a point, then $Y$ is bounded by Lemma 5.1. This shows (2) $\Leftrightarrow(3)$.

The implication $(3) \Rightarrow(4)$ is clear. For the converse, assume that $Y$ is an unbounded cylinder. We know that $G_{Y}$ does not fix a point. If all its elements are elliptic, then 
$G_{Y}$ fixes an end of $Y$. Any ray going out to that end maps injectively to $Y / G_{Y}$, contradicting Lemma 5.1. Thus $G_{Y}$ contains a hyperbolic element $g$. Being $g-$ invariant, $Y$ contains the axis of $g$.

Proposition 5.3 Assume that any two groups of $\mathcal{E}$ whose intersection is infinite are equivalent. Let $H$ be a subgroup of $G$ which is not virtually cyclic and is not an infinite, locally finite, torsion group.

If $H$ is small, or commensurates an infinite small subgroup $H_{0}$, then $H$ fixes a point in $T_{c}$.

The hypothesis on $\mathcal{E}$ is satisfied in Sections 3.1 to 3.6, with the exception of 3.2. Conversely, we will see in Section 6 that, in many examples, groups which are elliptic in $T_{c}$ but not in $T$ are small.

Besides small groups, the proposition applies to groups $H$ which act on locally finite trees with small infinite stabilizers, for instance generalized Baumslag-Solitar groups. It also applies to groups with a small infinite normal subgroup, such as fundamental groups of Seifert fibered spaces.

Proof The result is clear if $H$ fixes a point of $T$. If not, we show that $H$ preserves a subtree $Y_{0}$ contained in a cylinder. This cylinder will be $H$-invariant.

First suppose that $H$ is small. If $H$ preserves a line $\ell$, then $G_{e} \cap H$ is the same for all edges in that line. If $G_{e} \cap H$ is infinite, then $\ell$ is contained in a cylinder and one can take $Y_{0}=\ell$. If $G_{e} \cap H$ is finite, then $H$ is virtually cyclic, which is ruled out. By smallness, the only remaining possibility is that $H$ fixes a unique end of $T$.

If $G_{e} \cap H$ is infinite for some edge $e$, we let $\rho$ be the ray joining $e$ to the fixed end. The assumption on $\mathcal{E}$ implies that $\rho$ is contained in a cylinder $Y_{0}$. This cylinder is $H$-invariant since $h \rho \cap \rho$ is a ray for any $h \in H$.

If all groups $G_{e} \cap H$ are finite, there are two cases. If $H$ contains a hyperbolic element $h$, the action of $H$ on its minimal subtree $T_{H}$ is an ascending HNN extension with finite edge groups. It follows that $T_{H}$ is a line and $H$ is virtually cyclic. If every element of $H$ is elliptic, consider any finitely generated subgroup $H_{0} \subset H$. It fixes both an end and a point, so it fixes an edge. We conclude that $H_{0}$ is finite, so $H$ is locally finite. This is ruled out.

Now suppose that $H$ commensurates a small subgroup $H_{0}$. If $H_{0}$ preserves a unique line or fixes a unique end, the same is true for $H$ and we argue as before. If $H_{0}$ fixes a point $x \in T$, let $Y_{0}$ be the convex hull of the orbit $H . x$. Any segment $I \subset Y_{0}$ is contained in a segment $\left[h x, h^{\prime} x\right]$ with $h, h^{\prime} \in H$, and its stabilizer contains $h H_{0} h^{-1} \cap h^{\prime} H_{0} h^{-1}$ which is commensurable to $H_{0}$ hence infinite. The assumption on $\mathcal{E}$ implies that $Y_{0}$ is contained in a cylinder. 
Remark 5.4 Assume that there exists $C$ such that any two groups of $\mathcal{E}$ whose intersection has order $>C$ are equivalent. The same proof shows that locally finite subgroups are elliptic in $T_{c}$.

\subsection{The collapsed tree of cylinders $T_{c}^{*}$}

Recall that the collapsed tree of cylinders $T_{c}^{*}$ is the tree obtained from $T_{c}$ by collapsing all edges whose stabilizer is not in $\mathcal{E}$ (Definition 4.5).

Proposition 5.5 Cylinders of $T_{c}^{*}$ have diameter at most 2 .

Proof This follows from Remark 4.6: if in $\mathcal{E}$, the stabilizer of an edge $(x, Y)$ of $T_{c}$ belongs to the equivalence class $\mathcal{C}$ associated to $Y$.

We say that $\mathcal{E}$ is sandwich-closed if $A \subset H \subset B$ with $A, B \in \mathcal{E}$ implies $H \in \mathcal{E}$. All families considered in Sections 3.1 through 3.6 have this property.

Sandwich-closedness has the following consequence. If $\varepsilon$ is an edge of $T_{c}$ such that $G_{\varepsilon}$ is contained in a group of $\mathcal{E}$, then $G_{\mathcal{E}} \in \mathcal{E}$. This follows from Remark 4.4, which asserts that $G_{\varepsilon}$ contains a group of $\mathcal{E}$.

Lemma 5.6 Assume that $\mathcal{E}$ is sandwich-closed. Given an equivariant map $f: T \rightarrow T^{\prime}$, the cellular map $f_{c}: T_{c} \rightarrow T_{c}^{\prime}$ of Proposition 4.11 induces a cellular map $f_{c}^{*}: T_{c}^{*} \rightarrow T_{c}^{\prime *}$.

Proof If $\varepsilon$ is an edge of $T_{c}$ which is collapsed in $T_{c}^{*}$, its image by $f_{c}$ is a point or an edge $\varepsilon^{\prime}$ with $G_{\varepsilon} \subset G_{\varepsilon^{\prime}}$. The group $G_{\varepsilon}$ is not in $\mathcal{E}$, but by Remark 4.4 it contains an element of $\mathcal{E}$. Sandwich-closedness implies $G_{\mathcal{E}^{\prime}} \notin \mathcal{E}$, so $\varepsilon^{\prime}$ is collapsed in $T_{c}^{\prime *}$. This shows that the natural map $T_{c} \rightarrow T_{c}^{\prime *}$ factors through $T_{c}^{*}$.

A subtree $X \subset T$ of diameter exactly 2 has a center $v \in V(T)$, and all its edges contain $v$. We say that $X$ is complete if it contains all edges around $v$, incomplete otherwise.

Proposition 5.7 Assume that $\mathcal{E}$ is sandwich-closed. Let $T$ be a minimal $\mathcal{E}$-tree.

(1) Every cylinder of $T_{c}^{*}$ has diameter exactly 2. No stabilizer of an incomplete cylinder of $T_{c}^{*}$ lies in $\mathcal{E}$.

(2) Conversely, assume that all cylinders of $T$ have diameter exactly 2, and $G_{Y} \notin \mathcal{E}$ for all incomplete cylinders $Y \subset T$. Then $T_{c}^{*}=T$. 
Proof It follows from Proposition 5.5 that any cylinder $Z$ of $T_{c}^{*}$ has diameter at most 2, and is obtained from the ball of radius one around some $Y \in V_{1}\left(T_{c}\right)$ by collapsing all edges with stabilizer outside $\mathcal{E}$. It is incomplete if and only if at least one edge is collapsed. Note that the cylinders $Y \subset T$ and $Z \subset T_{c}^{*}$ have the same stabilizer $G_{Y}=G_{Z}$.

We show that $Z$ has diameter exactly 2 . Otherwise $Z$ consists of a single edge. The corresponding edge $\varepsilon=(v, Y)$ of $T_{c}$ is the unique edge incident on $Y$ with $G_{\varepsilon} \in \mathcal{E}$, so $G_{\varepsilon}=G_{Y}$ (otherwise, one would obtain other edges by applying elements of $G_{Y} \backslash G_{\varepsilon}$ ). By minimality of $T_{c}$, there exist other edges $\varepsilon^{\prime}$ incident on $Y$. They satisfy $G_{\mathcal{E}^{\prime}} \notin \mathcal{E}$, and $G_{\varepsilon^{\prime}} \subset G_{Y} \in \mathcal{E}$ contradicts sandwich-closedness.

If $Z$ is an incomplete cylinder of $T_{c}^{*}$, at least one edge $\varepsilon$ of $T_{c}$ incident on $Y$ is collapsed in $T_{c}^{*}$, so $G_{\varepsilon} \notin \mathcal{E}$. As above, sandwich-closedness implies $G_{Y} \notin \mathcal{E}$. This proves (1).

To prove (2), we shall define an isomorphism $g: T_{c}^{*} \rightarrow T$. We denote by $v_{Y}$ the center of a cylinder $Y$ of $T$. Let $f: T_{c} \rightarrow T$ be the map sending $Y \in V_{1}\left(T_{c}\right)$ to $v_{Y} \in T$, sending $v \in V_{0}\left(T_{c}\right)$ to $v \in T$, and mapping the edge $\varepsilon=(v, Y)$ to $\left[v, v_{Y}\right]$. Note that $\left[v, v_{Y}\right]$ is an edge if $v \neq v_{Y}$, and is reduced to a point otherwise.

We first prove that an edge $\varepsilon$ of $T_{c}$ is collapsed by $f$ (ie $v=v_{Y}$ ) if and only if $G_{\varepsilon} \notin \mathcal{E}$. If $G_{\varepsilon} \notin \mathcal{E}$, sandwich-closedness implies that $\varepsilon$ is collapsed, since $T$ is an $\mathcal{E}$-tree. Conversely, if $\varepsilon=(v, Y)$ is collapsed, then $v_{Y}=v \in V_{0}(T)$, so $v_{Y}$ lies in several cylinders of $T$. This implies that $Y$ is incomplete, so $G_{Y} \notin \mathcal{E}$. Since $G_{Y}$ is contained in $G_{v_{Y}}=G_{v}$, one has $G_{\varepsilon}=G_{Y} \notin \mathcal{E}$.

It follows that $f$ factors through a map $g: T_{c}^{*} \rightarrow T$ which maps edge to edge (without collapse). By minimality, $g$ is onto. There remains to prove that $g$ does not fold. If two edges of $T_{c}^{*}$ have the same image, they belong to the same cylinder. But $g$ is injective on each cylinder since $(v, Y)$ is mapped to $\left[v, v_{Y}\right]$.

Corollary 5.8 Let $\mathcal{E}$ be sandwich-closed. For any minimal $\mathcal{E}$-tree $T$, one has $\left(T_{c}^{*}\right)_{c}^{*}=T_{c}^{*}$.

Proposition 5.9 Assume that $G_{Y}$ fixes a point of $T$ whenever there is an edge $\varepsilon=(x, Y)$ of $T_{c}$ whose stabilizer is not in $\mathcal{E}$. Then $T_{c}$ and $T_{c}^{*}$ belong to the same deformation space. Moreover, given $Y$, at most one edge $\varepsilon=(x, Y)$ of $T_{c}$ is collapsed in $T_{c}^{*}$; it satisfies $G_{\varepsilon}=G_{Y}$.

Proof Let $\varepsilon=(x, Y)$ be an edge of $T_{c}$ such that $G_{\varepsilon} \notin \mathcal{E}$. It suffices to prove that $G_{\varepsilon}=G_{Y}$ and that $G_{\mathcal{E}^{\prime}} \in \mathcal{E}$ for every edge $\varepsilon^{\prime}=\left(x^{\prime}, Y\right)$ with $x^{\prime} \neq x$. 
By assumption, $G_{Y}$ fixes a point in $T$, hence a point $v \in Y$. If $x \neq v$, let $e$ be the initial edge of the segment $[x, v]$. Then $G_{\varepsilon}=G_{x} \cap G_{Y} \subset G_{X} \cap G_{v} \subset G_{e}$. On the other hand, $G_{e}$ fixes $x$ and leaves $Y$ invariant, so $G_{e} \subset G_{\varepsilon}$. We conclude $G_{\varepsilon}=G_{e} \in \mathcal{E}$, a contradiction. Thus $x=v$, and $\varepsilon=(x, Y)$ is the only edge incident to $Y$ with stabilizer not in $\mathcal{E}$. Moreover, since $G_{Y}$ fixes $x$, we have $G_{\varepsilon}=G_{Y} \cap G_{x}=G_{Y}$.

Corollary 5.10 If $T_{c}$ is in the same deformation space as $T$, then so is $T_{c}^{*}$, and therefore $\left(T_{c}^{*}\right)_{c}=T_{c}$ by Corollary 4.10 .

Remark 5.11 Suppose that $\mathcal{E}$ is sandwich-closed and that, for any $A \in \mathcal{E}$, any subgroup containing $A$ with index 2 lies in $\mathcal{E}$. Then the hypothesis of Proposition 5.9 is always satisfied when $G_{Y}$ is small. To see this, we suppose that $G_{Y}$ is not elliptic in $T$ and we show $G_{\varepsilon} \in \mathcal{E}$. If $G_{Y}$ fixes an end of $T$, its subgroup $G_{\mathcal{\varepsilon}}$, being elliptic, fixes an edge. Since $G_{\varepsilon}$ contains a group in $\mathcal{E}$, sandwich-closedness implies $G_{\varepsilon} \in \mathcal{E}$. If $G_{Y}$ acts dihedrally on a line, some subgroup of $G_{\varepsilon}$ of index at most 2 fixes an edge, so $G_{\varepsilon} \in \mathcal{E}$.

Recall that cylinders of $T_{c}^{*}$ have diameter at most 2 . We show that $T_{c}^{*}$ is maximal for this property.

Proposition 5.12 Assume that $\mathcal{E}$ is sandwich-closed. If $T^{\prime}$ is any $\mathcal{E}$-tree dominated by $T$, and cylinders of $T^{\prime}$ are bounded, then $T^{\prime}$ is dominated by $T_{c}^{*}$.

Proof By Proposition 5.2 and Corollary 5.10, the tree $T_{c}^{\prime *}$ belongs to the same deformation space as $T^{\prime}$. Lemma 5.6 shows that $T_{c}^{*}$ dominates $T_{c}^{\prime *}$, hence $T^{\prime}$.

\subsection{Acylindricity}

We now consider acylindricity in the sense of Sela. Recall [27] that a tree is $k-$ acylindrical if the stabilizer of any segment of length $>k$ is trivial. It is acylindrical if it is $k$-acylindrical for some $k$. To handle groups with torsion, we say that $T$ is almost $k$-acylindrical if the stabilizer of any segment of length $>k$ is finite.

Proposition 5.13 Assume that any two groups of $\mathcal{E}$ whose intersection is infinite are equivalent. Let $T$ be any $\mathcal{E}$-tree.

(1) The tree $T_{c}^{*}$ is almost 2-acylindrical.

(2) If cylinders of $T$ are bounded (resp. of diameter $\leq k$ ), then $T$ is almost acylindrical (resp. almost $k$-acylindrical). 
Recall that the hypothesis on $\mathcal{E}$ is satisfied in Sections 3.1 to 3.6, with the exception of 3.2. The next section will provide examples where the converse to Assertion (2) holds.

Proof The first statement follows from the second one and Proposition 5.5.

Since there are only finitely many orbits of cylinders, consider $k$ such that cylinders have diameter at most $k$. Any segment $I$ of length $k+1$ contains edges in distinct cylinders. By the assumption on $\mathcal{E}$, the stabilizer of $I$ is finite.

We also note:

Lemma 5.14 Let $H$ be small, not virtually cyclic, not locally finite. Then $H$ is elliptic in any almost acylindrical tree $T$.

Proof The hypotheses on $H$ are the same as in Proposition 5.3, and the proof is similar. If $H$ is not elliptic in a tree $T$, it acts on a line with infinite edge stabilizers, or it fixes a unique end and some edge stabilizer is infinite. Both are impossible if $T$ is almost acylindrical.

\section{Examples}

We now study specific examples. In most cases, we show that $T_{c}^{*}$ is equal to $T_{c}$ (or at least in the same deformation space), and we describe how far the deformation space of $T_{c}$ is from that of $T$.

Recall that $T$ always dominates $T_{c}$. They are in the same deformation space if and only if, for every cylinder $Y$, the group $G_{Y}$ is elliptic in $T$. Note that, if all groups in $\mathcal{E}$ are infinite, any virtually cyclic $G_{Y}$ is elliptic in $T$ because by Remark 4.4 it contains some $G_{e}$ (with finite index).

We also show that $T_{c}^{*}$, which is almost 2-acylindrical by Proposition 5.13, is maximal for this property: it dominates any almost acylindrical tree which is dominated by $T$. This is because, in the examples, groups which are elliptic in $T_{c}^{*}$ but not in $T$ are small, and Lemma 5.14 applies. Describing the deformation space of $T_{c}^{*}$ may thus be interpreted as finding which subgroups must be made elliptic in order to make $T$ almost acylindrical. One may ask in general whether a maximal almost acylindrical tree dominated by a given $T$ always exists. 


\subsection{Relatively hyperbolic groups}

Proposition 6.1 Let $G$ be hyperbolic relative to $H_{1}, \ldots, H_{n}$. Let $\sim$ be coelementarity, as in Section 3.3. Let $T$ be a tree with infinite elementary edge stabilizers, such that each $H_{i}$ is elliptic in $T$.

(1) Edge stabilizers of $T_{c}$ are infinite elementary, so $T_{c}^{*}=T_{c}$.

(2) $T_{c}$ belongs to the same deformation space as $T$. In particular, it has the same nonelementary vertex stabilizers as $T$.

(3) $T_{c}$ is almost 2-acylindrical (and dominates any almost acylindrical tree which is dominated by $T$ ).

Proof Let $\varepsilon=(x, Y)$ be an edge of $T_{c}$. Here $G_{Y}$ is the maximal elementary subgroup containing $G_{e}$, for any edge $e$ of $Y$. This shows that $G_{\varepsilon}$ is elementary. It is infinite because it contains an element of $\mathcal{E}$ (Remark 4.4).

To prove (2), we must show that every $G_{Y}$ is elliptic in $T$. If parabolic, $G_{Y}$ is elliptic by assumption. If virtually cyclic, it is elliptic by a remark made above (it contains some $G_{e}$ with finite index). Assertion (2) follows (its second half is a general fact [14, Corollary 4.4]).

Assertion (3) now follows from Proposition 5.13 (the parenthesized statement is trivial in this case).

If we do not assume that $H_{i}$ is elliptic in $T$, we get:

Proposition 6.2 Let $G$ be hyperbolic relative to finitely generated one-ended subgroups $H_{1}, \ldots, H_{n}$. Let $\sim$ be coelementarity, as in Section 3.4. Let $T$ be a tree with infinite elementary edge stabilizers.

(1) Edge stabilizers of $T_{c}$ are infinite elementary, so $T_{c}^{*}=T_{c}$.

(2) $T$ and $T_{c}$ have the same nonelementary vertex stabilizers. A subgroup is elliptic in $T_{c}$ if and only if it is elliptic in $T$, or parabolic. In particular, $T_{c}$ is in the same deformation space as $T$ if and only if every parabolic subgroup is elliptic in $T$.

(3) $T_{c}$ is almost 2-acylindrical. If the $H_{i}$ 's are small, then $T_{c}$ dominates any almost acylindrical tree $T^{\prime}$ which is dominated by $T$.

Proof It is still true that every $G_{Y}$ is a maximal elementary subgroup (so (1) holds), but a parabolic $G_{Y}$ may now fail to be elliptic in $T$. As pointed out at the beginning of the section, every virtually cyclic $G_{Y}$ is elliptic in $T$. 
If $G_{v}$ is a nonelementary vertex stabilizer of $T$, then $v$ belongs to two cylinders (otherwise $G_{v}$ would be contained in some $G_{Y}$ ), so $G_{v}$ is a vertex stabilizer of $T_{c}$. The converse is clear since a nonelementary vertex stabilizer of $T_{c}$ fixes a vertex of $V_{0}\left(T_{c}\right)$, so is a vertex stabilizer of $T$.

To prove (2), there remains to show that each $H_{i}$ is elliptic in $T_{c}$. If it is not elliptic in $T$, there is an edge $e$ with $G_{e} \cap H_{i}$ infinite (recall that $H_{i}$ is one-ended). In particular, $G_{e} \sim H_{i}$. The associated equivalence class $\mathcal{C}$ is invariant under conjugation by elements of $H_{i}$, so $H_{i}$ preserves the cylinder containing $e$ hence is elliptic in $T_{c}$. Acylindricity again follows from Proposition 5.13. The second part of (3) holds provided every $H_{i}$ is elliptic in $T^{\prime}$, in particular if $H_{i}$ is small by Lemma 5.14.

\subsection{Abelian splittings of CSA groups}

Proposition 6.3 Let $G$ be a torsion-free CSA group. Let $\mathcal{E}$ (nontrivial abelian groups) and $\sim$ (commutation) be as in Section 3.5. Let $T$ be an $\mathcal{E}$-tree.

(1) Edge stabilizers of $T_{c}$ are nontrivial and abelian, so $T_{c}^{*}=T_{c}$.

(2) $T$ and $T_{c}$ have the same nonabelian vertex stabilizers. A subgroup is elliptic in $T_{c}$ if and only if it is elliptic in $T$ or is a noncyclic abelian group. In particular, $T_{c}$ is in the same deformation space as $T$ if and only if every noncyclic abelian subgroup of $G$ is elliptic in $T$.

(3) $T_{c}$ is 2-acylindrical and dominates any acylindrical $\mathcal{E}$-tree $T^{\prime}$ which is dominated by $T$.

Proof If $Y \in V_{1}\left(T_{c}\right)$ is a cylinder, its stabilizer $G_{Y}$ is the set of $g \in G$ such that $g G_{e} g^{-1}$ commutes with $G_{e}$, for $e$ any edge of $Y$. By the CSA property, $G_{Y}$ is the maximal abelian subgroup containing $G_{e}$ (if $g \in G_{Y}$, then $g G_{e} g^{-1}$ and $G_{e}$ are contained in the same maximal abelian subgroup $A$, and $g \in A$ by malnormality). Conversely, a noncyclic abelian subgroup acts on $T$ with nontrivial edge stabilizers and therefore leaves some cylinder invariant. As in the previous proof, nonabelian vertex stabilizers are the same for $T$ and $T_{c}$.

Assertions (1) and (2) follow from these observations. The vertex stabilizers of $T_{c}$ are the nonabelian vertex stabilizers of $T$, the noncyclic maximal abelian subgroups, and possibly cyclic subgroups which are elliptic in $T$.

The tree $T_{c}$ is 2-acylindrical by Proposition 5.13. A group $H$ which is elliptic in $T_{c}$ but not in $T$ is abelian and noncyclic, hence elliptic in $T^{\prime}$ by Lemma 5.14. Assertion (3) follows.

We also note the following result, which gives a converse to the second assertion of Proposition 5.13: 
Proposition 6.4 Let $G, \mathcal{E}$ and $T$ be as in Proposition 6.3. The following are equivalent:

(1) Every noncyclic abelian subgroup is elliptic.

(2) Cylinders of $T$ are bounded (equivalently, $T_{c}$ is in the same deformation space as $T)$.

(3) $T$ is acylindrical.

(4) No nontrivial element of $G$ fixes a line.

Proof We have just seen $(1) \Leftrightarrow(2)$. Proposition 5.13 gives $(2) \Rightarrow(3)$, and obviously $(3) \Rightarrow(4)$. To prove $(4) \Rightarrow(2)$, suppose that $Y$ is an unbounded cylinder. By Proposition 5.2, it contains the axis of a hyperbolic element $g$. Let $e$ be an edge contained in that axis, and $A$ the maximal abelian subgroup containing $G_{e}$. Since $g G_{e} g^{-1}$ commutes with $G_{e}$, the CSA property implies $g \in A$. Thus $G_{e}$ fixes the axis, contradicting (4).

\subsection{Cyclic splittings of commutative transitive groups}

The relation $\sim$ now is commensurability, as in Section 3.1, so $G_{Y}$ is the commensurator of $G_{e}$ if $e$ is an edge of a cylinder $Y$.

For $s \neq 0$, denote by BS $(1, s)$ the solvable Baumslag-Solitar group $\left\langle a, t \mid t a t^{-1}=a^{s}\right\rangle$. It is commutative transitive if and only if $s \neq-1$. Note that $\operatorname{BS}(1,1)=\mathbb{Z}^{2}$.

Proposition 6.5 Let $G$ be torsion-free and commutative transitive. Let $\mathcal{E}$ be the class of infinite cyclic subgroups of $G$, and let $\sim$ be commensurability as in Section 3.1. Let $T$ be an $\mathcal{E}$-tree.

(1) $T_{c}^{*}$ and $T_{c}$ are in the same deformation space.

(2) Every noncyclic vertex stabilizer of $T$ is a vertex stabilizer of $T_{c}$ and $T_{c}^{*}$, and every other noncyclic vertex stabilizer of $T_{c}^{*}$ is isomorphic to some $\operatorname{BS}(1, s)$. Every subgroup isomorphic to $\mathrm{BS}(1, s)$ is elliptic in $T_{c}$ and $T_{c}^{*}$. In particular, $T_{c}$ and $T_{c}^{*}$ belong to the same deformation space as $T$ if and only if every subgroup of $G$ isomorphic to a $\mathrm{BS}(1, s)$ is elliptic in $T$.

(3) $T_{c}^{*}$ is 2 -acylindrical and dominates any acylindrical $\mathcal{E}$-tree which is dominated by $T$.

Proof We note the following algebraic facts, whose proof is left to the reader. Let $Z \subset H$ be an infinite cyclic subgroup of a commutative transitive torsion-free group, and let $A$ be the centralizer of $Z$. Then $Z \subset A \subset \operatorname{Comm}(Z) \subset N(A)$. If $A=Z$, then $Z$ is malnormal. 
Consider a cylinder $Y \subset T$, and a vertex $v \in Y$. All edge stabilizers $G_{e}$, for $e \subset Y$, are commensurable, hence have the same centralizer $A$ by commutative transitivity. By the previous remark, one has $A \subset G_{Y} \subset N(A)$ since $G_{Y}$ is the commensurator of $G_{e}$.

Lemma 6.6 Assume that $G_{v} \cap G_{Y}$ is noncyclic. Then $G_{Y}$ fixes $v$, and only $v$. Moreover,

(1) if $Y$ is the only cylinder containing $v$, then $G_{Y}=G_{v}$ and no edge of $T_{c}$ incident to the vertex $Y \in V_{1}\left(T_{c}\right)$ gets collapsed in $T_{c}^{*}$;

(2) if $v$ belongs to two cylinders, the edge $\varepsilon=(v, Y)$ of $T_{c}$ is collapsed in $T_{c}^{*}$ (the vertex $Y$ "disappears" in $T_{c}^{*}$ ).

Proof We first show that $G_{v} \cap A$ is noncyclic. Assume that $G_{v} \cap A$ is cyclic, necessarily infinite since it contains $G_{e}$ for $e$ an edge of $Y$ adjacent to $v$. By the initial note above, $G_{v} \cap A$ is malnormal in $G_{v}$, so $G_{v} \cap G_{Y}=G_{v} \cap A$ is cyclic, a contradiction.

Since $G_{v} \cap A$ is noncyclic, $v$ is its unique fixed point. It is also the unique fixed point of $A$ (which centralizes $G_{v} \cap A$ ), and of $G_{Y} \subset N(A)$.

The "moreover" is clear: the only collapsible edge of $T_{c}$ incident to $Y$ is $(v, Y)$, which exists if and only if $v$ belongs to two cylinders.

By Proposition 5.9, the lemma implies that $T_{c}$ and $T_{c}^{*}$ belong to the same deformation space. Moreover, any vertex stabilizer $H$ of $T_{c}^{*}$ which is not a vertex stabilizer of $T$ equals $G_{Y}$ for some cylinder $Y$ such that $G_{v} \cap G_{Y}$ is cyclic for every vertex $v \in Y$. The group $G_{Y}$ acts on $Y$ with all edge and vertex stabilizers infinite cyclic. Since it is commutative transitive, it is easy to see that $G_{Y}$ must be isomorphic to $\mathbb{Z}$ or a $\mathrm{BS}(1, s)$ (otherwise $G_{Y}$ contains $F_{2} \times \mathbb{Z}$ or $\left\langle a, b \mid a^{m}=b^{n}\right\rangle$ with $m, n \geq 2$; such groups are not commutative transitive). Conversely, any $\mathrm{BS}(1, s)$ is elliptic in $T_{c}$ by Proposition 5.3.

To prove Assertion (2), there remains to show that any noncyclic vertex stabilizer $G_{v}$ of $T$ is a vertex stabilizer of $T_{c}$ and $T_{c}^{*}$. This is clear if $v$ belongs to two cylinders. If it belongs to a unique cylinder $Y$, the lemma tells us that $G_{v}=G_{Y}$ is a vertex stabilizer of $T_{c}$ and of $T_{c}^{*}$.

Assertion (3) now follows from Proposition 5.13 and Lemma 5.14.

\subsection{Commensurability}

In our last examples $\sim$ is again commensurability, but we do not make assumptions on $G$, so our results are less precise. 
Proposition 6.7 Let $\mathcal{E}$ be the set of two-ended subgroups of $G$, and $\sim$ be commensurability, as in Section 3.1. Given an $\mathcal{E}$-tree $T$, the following are equivalent:

(1) Cylinders of $T$ are bounded (equivalently, $T_{c}$ is in the same deformation space as $T$ ).

(2) The commensurator of each edge stabilizer is elliptic in $T$.

(3) $T$ is almost acylindrical.

(4) No element of infinite order fixes a ray.

Proof $(1) \Leftrightarrow(2)$ is clear because $G_{Y}=\operatorname{Comm}\left(G_{e}\right)$ if $e \subset Y$. (1) $\Rightarrow$ (3) follows from Proposition 5.13 and $(3) \Rightarrow(4)$ is clear.

To prove $(4) \Rightarrow(1)$, assume that some cylinder is unbounded. By Proposition 5.2, it contains the axis $A_{g}$ of a hyperbolic element $g$. Let $e_{0}$ be an edge of $A_{g}$, let $e_{i}=$ $g^{i}\left(e_{0}\right)$, and $H_{i}=G_{e_{i}}$. If $H_{i} \subset H_{i \pm 1}$ for some $i$, then $H_{i}$ fixes a ray, contradicting (4). If not, we can find $h_{0} \in H_{0}$ and $h_{2} \in H_{2}$ not fixing $e_{1}$, and $h=h_{0} h_{2}$ is hyperbolic. As $H_{0}$ and $H_{2}$ are commensurable, there is a finite index subgroup $H \subset H_{0} \cap H_{2}$ which is normal in both $\mathrm{H}_{0}$ and $\mathrm{H}_{2}$. Since $h$ normalizes $H$, the fixed point set of $H$ contains the axis of $h$, and (4) does not hold.

Corollary 6.8 For any $\mathcal{E}$-tree $T$, the tree $T_{c}^{*}$ is almost 2-acylindrical and dominates any almost acylindrical $\mathcal{E}$-tree which is dominated by $T$.

Proof This follows from Proposition 5.12 and Proposition 5.13.

In Section 3.2, we get:

Proposition 6.9 Let $\sim$ be the commensurability relation, with $\mathcal{E}$ as in Section 3.2. Let $T$ be an $\mathcal{E}$-tree.

(1) $T_{c}$ belongs to the same deformation space as $T$ if and only if there exists $k$ such that any segment $I$ of length $>k$ contains an edge $e$ with the index $\left[G_{e}: G_{I}\right]$ infinite.

(2) Assume that edge stabilizers of $T$ are finitely generated. Then $T_{c}$ belongs to the same deformation space as $T$ if and only if no group commensurable to an edge stabilizer fixes a ray.

In particular, $T_{c}$ belongs to the same deformation space as $T$ when every group in $\mathcal{E}$ is infinite and $T$ is almost acylindrical. But without further hypotheses on $\mathcal{E}$ we cannot claim that $T_{c}^{*}$ is almost acylindrical. 
Proof (1) follows from the fact that a segment $I$ is contained in a cylinder if and only if $\left[G_{e}: G_{I}\right]$ is finite for every $e \subset I$. The proof of (2) is fairly similar to that of Proposition 6.7, and left to the reader. Finite generation of edge stabilizers is used to construct the normal subgroup of finite index $H$.

\section{JSJ splittings}

\subsection{Generalities}

We review basic facts about JSJ splittings and JSJ deformation spaces. See our papers $[15 ; 17]$ for details.

In order to define JSJ splittings, one needs a family of edge groups which is closed under taking subgroups. Since $\mathcal{E}$ does not always have this property, we introduce the following substitute.

Definition 7.1 The family $\mathcal{E}$ is substable if, whenever $G$ splits over a group $A$ contained in a group $B \in \mathcal{E}$, then $A \in \mathcal{E}$.

Remark 7.2 When we work relative to a family of subgroups (like in Section 3.3), the splitting of $G$ in the definition should be relative to this family.

Example 7.3 In Sections 3.1, 3.4, 3.5 (with $G$ torsion-free), $\mathcal{E}$ is substable if and only if $G$ is one-ended. In Section 3.2, $\mathcal{E}$ is substable if and only if $G$ does not split over a group having infinite index in a group of $\mathcal{E}$. In Section 3.3, we restrict to relative splittings, and $\mathcal{E}$ is substable if and only if $G$ is one-ended relative to the $H_{i}$ 's (ie there is no nontrivial tree with finite edge stabilizers in which every $H_{i}$ is elliptic).

We fix $\mathcal{E}$ and $\sim$, with $\mathcal{E}$ substable. All trees are assumed to be $\mathcal{E}$-trees. Strictly speaking, we consider $\overline{\mathcal{E}}$-trees, where $\overline{\mathcal{E}}$ consists of all groups contained in a group of $\mathcal{E}$. But substability guarantees that every $\overline{\mathcal{E}}$-tree is an $\mathcal{E}$-tree.

A subgroup $H \subset G$ is universally elliptic if it is elliptic in every tree. A tree is universally elliptic if all its edge stabilizers are.

A tree is a JSJ tree over $\mathcal{E}$ if it is universally elliptic, and maximal for this property: it dominates every universally elliptic tree. When $\overline{\mathcal{E}}$ consists of all groups with a given property (eg abelian, slender, elementary), we use the words abelian JSJ, slender JSJ, elementary JSJ....

JSJ trees always exist when $G$ is finitely presented, and sometimes when $G$ is only finitely generated (in particular in the situations studied below). They belong to the 
same deformation space, called the JSJ deformation space over $\mathcal{E}$. If $T_{J}$ is a JSJ tree, and $T^{\prime}$ is any tree, there is a tree $\widehat{T}$ which refines $T_{J}$ and dominates $T^{\prime}$.

All these definitions and facts extend to the relative case: given a collection of subgroups, one only considers trees in which these subgroups are elliptic.

\subsection{QH-vertices}

A vertex stabilizer of a JSJ tree is flexible if it is not universally elliptic, and does not belong to $\overline{\mathcal{E}}$. A key fact of JSJ theory is that flexible vertex stabilizers often have a very special form.

Definition 7.4 A vertex stabilizer $G_{v}$ is a $\mathrm{QH}$-subgroup (and $v$ is a $\mathrm{QH}$-vertex) if there is an exact sequence $1 \rightarrow F \rightarrow G_{v} \stackrel{\pi}{\rightarrow} \Sigma \rightarrow 1$, where $\Sigma=\pi_{1}(S)$ is the fundamental group of a hyperbolic 2-orbifold with boundary. Moreover, each incident edge stabilizer is conjugate to a subgroup of a boundary subgroup $B \subset G_{v}$, defined as the preimage under $\pi$ of $\pi_{1}(C)$, with $C$ a component of $\partial S$.

In Section 8.3 we will need a description of flexible vertex stabilizers in the following cases (see [18, Sections 11 and 13] for proofs). Assume that $G$ is one-ended.

- $G$ is torsion-free and CSA. A flexible vertex stabilizer $G_{v}$ of an abelian JSJ tree is a QH-subgroup, with $S$ a surface and $F$ trivial: $G_{v}$ is isomorphic to $\Sigma=\pi_{1}(S)$, where $S$ is a compact surface.

- $G$ is hyperbolic relative to slender subgroups. A flexible vertex stabilizer $G_{v}$ of a slender JSJ tree is a QH-subgroup, with $F$ finite.

In both cases, every incident edge stabilizer is conjugate to a finite index subgroup of a boundary subgroup. Boundary subgroups are two-ended, and maximal among small subgroups of $G_{v}$. Every boundary subgroup contains an incident edge stabilizer.

\subsection{Canonical JSJ splittings}

We now use trees of cylinders to make JSJ splittings canonical (ie we get trees which are invariant under automorphisms). See [18] for a proof that JSJ splittings exist under the stated hypotheses, and a discussion of their flexible vertices.

Theorem 7.5 Let $G$ be hyperbolic relative to $H_{1}, \ldots, H_{n}$. Assume that $G$ is oneended relative to $H_{1}, \ldots, H_{n}$. There is an elementary (resp. virtually cyclic) JSJ tree relative to $H_{1}, \ldots, H_{n}$ which is invariant under the subgroup of $\operatorname{Out}(G)$ preserving the conjugacy classes of the $H_{i}$ 's. 
Remark 7.6 When $H_{i}$ is not slender, this allows nonslender splittings. Still, one can describe flexible subgroups of this JSJ tree as QH-subgroups [18, Section 13].

Proof First consider the case where $\mathcal{E}$ is the family of infinite elementary subgroups, as in Section 3.3. It is substable because $G$ is one-ended relative to the $H_{i}$ 's (see Example 7.3). Let $T$ be an elementary JSJ tree relative to the $H_{i}$ 's, and $T_{c}$ its tree of cylinders for coelementarity.

By Proposition 6.1, the tree $T_{c}$ has elementary edge stabilizers and lies in the JSJ deformation space. It is universally elliptic as its edge stabilizers are either parabolic, or are virtually cyclic and contain an edge stabilizer of $T$ with finite index (Remark 4.4). It is invariant under the subgroup of $\operatorname{Out}(G)$ preserving the conjugacy classes of the $H_{i}$ 's because the JSJ deformation space is. The theorem follows.

Now turn to the case where $\mathcal{E}$ is the class of infinite virtually cyclic subgroups, still substable because of one-endedness. We start with a virtually cyclic JSJ tree (relative to the $H_{i}$ 's), we let $T_{c}$ be its tree of cylinders (for coelementarity, restricted to $\mathcal{E}$, not commensurability), and we consider $T_{c}^{*}$ obtained by collapsing edges of $T_{c}$ whose stabilizer is not virtually cyclic. By Proposition 5.9 and Proposition 6.1, the trees $T_{c}^{*}, T_{c}$ and $T$ lie in the same deformation space. Moreover, $T_{c}^{*}$ is universally elliptic because its edge stabilizers contain an edge stabilizer of $T$ with finite index (Remark 4.4). It follows that $T_{c}^{*}$ is a canonical JSJ splitting.

A similar argument, using Proposition 6.3, shows:

Theorem 7.7 Let $G$ be a one-ended torsion-free CSA group. There exists an abelian (resp. cyclic) JSJ tree of $G$ relative to all noncyclic abelian subgroups, which is $\operatorname{Out}(G)$ invariant.

\section{Compatibility}

Recall that two trees $T$ and $T^{\prime}$ are compatible if they have a common refinement. The goal of this section is to show that $T_{c}$ is compatible with many splittings. In particular, we show that trees of cylinders of JSJ deformation spaces often are universally compatible, that is compatible with every $\mathcal{E}$-tree.

This is proved under two different types of hypotheses: in Section 8.2 we assume that $\sim$ preserves universal ellipticity (this is true in particular when $\sim$ is commensurability), and in Section 8.3 we work in the setting of CSA groups and relative hyperbolic groups. Theorem 6 follows from Corollary 8.4 and Theorem 8.6 


\subsection{A general compatibility statement}

We first prove a general compatibility statement, independent of JSJ theory.

Proposition 8.1 Let $T, T^{\prime}$ be minimal $\mathcal{E}$-trees. If $T$ dominates $T^{\prime}$, then $T_{c}$ is compatible with $T^{\prime}$ and $T_{c}^{\prime}$.

Proof We have to construct a common refinement $\widehat{T}$ of $T_{c}$ and $T^{\prime}$ (compatibility of $T_{c}$ with $T_{c}^{\prime}$ will follow, by the proposition, since $T$ dominates $T_{c}^{\prime}$ ). Choose a map $f: T \rightarrow T^{\prime}$ as in Section 4.3. For each $p \in V\left(T_{c}\right)$, denote by $Y_{p}$ the following subset of $T$ : the point $p$ if $p \in V_{0}\left(T_{c}\right)$, the cylinder defining $p$ if $p \in V_{1}\left(T_{c}\right)$. Consider $Z_{p}=f\left(Y_{p}\right) \subset T^{\prime}$. By Lemma 4.12, it is either a point or a cylinder of $T^{\prime}$. Note that a given edge of $T^{\prime}$ is contained in exactly one $Z_{p}$.

We obtain $\widehat{T}$ from $T_{c}$ by "blowing up" each vertex $p$ to the subtree $Z_{p}$. Formally, we define $\hat{T}$ as the tree obtained from $T_{1}=\bigsqcup_{p \in V\left(T_{c}\right)} Z_{p}$ as follows: for each edge $p q$ of $T_{c}$, with $Y_{p}=\{x\}$ and $Y_{q}$ a cylinder containing $x$, add an edge to $T_{1}$, the endpoints being attached to the two copies of $f(x)$ in $Z_{p}$ and $Z_{q}$ respectively.

The tree $T_{c}$ can be recovered from $\hat{T}$ by collapsing each $Z_{p}$ to a point. We show that $\widehat{T}$ is also a refinement of $T^{\prime}$. Let $g: \hat{T} \rightarrow T^{\prime}$ be the map defined as being induced by the identity on $T_{1}$, and being constant on each added edge. It preserves alignment because a given edge of $T^{\prime}$ is contained in exactly one $Z_{p}$, and $g$ is injective on each $Z_{p}$. One therefore recovers $T^{\prime}$ from $\hat{T}$ by collapsing the added edges.

\subsection{Universal compatibility when $\sim$ preserves universal ellipticity}

As before, we fix $\mathcal{E}$ and $\sim$, with $\mathcal{E}$ substable. All trees are assumed to be $\mathcal{E}$-trees. In this subsection, we assume that $\sim$ preserves universal ellipticity in the following sense.

Definition 8.2 The relation $\sim$ preserves universal ellipticity if, given $A, B \in \mathcal{E}$ with $A \sim B$, the group $A$ is universally elliptic if and only if $B$ is.

For instance, commensurability always preserves universal ellipticity. In the case of a relatively hyperbolic group $G$, coelementarity preserves universal ellipticity if one restricts to trees in which each $H_{i}$ is elliptic (Section 3.3). Similarly, in Section 3.5, one has to restrict to trees in which noncyclic abelian subgroups are elliptic (the next subsection will provide nonrelative results).

Proposition 8.3 Assume that $\mathcal{E}$ is substable and $\sim$ preserves universal ellipticity. If $T_{J}$ is a JSJ tree over $\mathcal{E}$, its tree of cylinders is compatible with any $\mathcal{E}$-tree. 
Using Example 7.3, we immediately deduce:

Corollary 8.4 Let $G$ be finitely presented.

(1) Let $\mathcal{E}$ be the class of two-ended subgroups as in Section 3.1. If $G$ is one-ended, the tree of cylinders of the JSJ deformation space over $\mathcal{E}$ is compatible with any tree with two-ended edge stabilizers.

(2) More generally, let $\mathcal{E}$ and $\sim$ be as in Section 3.2. If $G$ does not split over a subgroup having infinite index in a group of $\mathcal{E}$, the tree of cylinders of the JSJ deformation space over $\mathcal{E}$ is compatible with any $\mathcal{E}$-tree.

(3) Let $G$ be hyperbolic relative to finitely generated subgroups $H_{i}$ as in Section 3.3, and assume that $G$ is one-ended relative to the $H_{i}$ 's. Then the tree of cylinders of the elementary JSJ deformation space relative to the $H_{i}$ 's is compatible with any $\mathcal{E}$-tree in which each $H_{i}$ is elliptic.

Remark 8.5 Finite presentability of $G$ is required only to know that the JSJ deformation space exists.

Proof of Proposition 8.3 Let $T$ be an $\mathcal{E}$-tree, and let $\widehat{T}$ be a refinement of $T_{J}$ which dominates $T$ (see Section 7.1). Let $X$ be the tree obtained from $\hat{T}$ by collapsing all the edges whose stabilizer is not $\sim$-equivalent to an edge stabilizer of $T_{J}$. The collapse map from $\hat{T}$ to $T_{J}$ factors through the collapse map $p: \hat{T} \rightarrow X$. In particular, $X$ dominates $T_{J}$.

Since $T_{J}$ is universally elliptic, and $\sim$ preserves universal ellipticity, $X$ is universally elliptic. By maximality of the JSJ deformation space, $X$ lies in the JSJ deformation space. In particular, $X$ and $T_{J}$ have the same tree of cylinders $X_{c}$. We have to show that $X_{c}$ is compatible with $T$.

Let $\widehat{T}_{c}$ be the tree of cylinders of $\widehat{T}$, which is compatible with $T$ by Proposition 8.1 since $\widehat{T}$ dominates $T$. Because of the way $X$ was defined, the restriction of $p: \hat{T} \rightarrow X$ to any cylinder is either constant or injective. By Remark 4.13, $p_{c}: \widehat{T}_{c} \rightarrow X_{c}$ is a collapse map, so $X_{c}$ is compatible with $T$.

\subsection{Universal compatibility when $\sim$ does not preserve universal ellipticity}

Theorem 8.6 Let $G$ be one-ended.

(1) Suppose $G$ is hyperbolic relative to slender subgroups $H_{1}, \ldots, H_{n}$. The tree of cylinders of the slender JSJ deformation space is compatible with every tree whose edge stabilizers are slender. 
(2) Suppose $G$ is torsion free and CSA. The tree of cylinders of the abelian JSJ deformation space is compatible with every tree whose edge stabilizers are abelian.

The tree of cylinders is defined with $\mathcal{E}$ as in Sections 3.4 and 3.5: it consists of all infinite slender (resp. abelian) subgroups, and $\sim$ is coelementarity (=co-slenderness) or commutation (note that each $H_{i}$ is finitely-ended, so $\mathcal{E}$ is admissible by Lemma 3.5). The family $\mathcal{E}$ is substable because $G$ is one-ended, but $\sim$ does not preserve universal ellipticity.

Proof Let $T_{J}$ be a JSJ tree, and $T_{c}$ its tree of cylinders. If $x \in V_{0}\left(T_{c}\right)$, we know that $G_{x} \notin \mathcal{E}$ (see Section 4.2). On the other hand $G_{Y} \in \mathcal{E}$ if $Y \in V_{1}\left(T_{c}\right)$, and edge stabilizers of $T_{c}$ belong to $\mathcal{E}$ (see Section 6.1 and Section 6.2).

We now show that $T_{c}$ is universally elliptic. Let $\varepsilon=(x, Y)$ be an edge. Let $e \subset Y$ be an edge of $T_{J}$ adjacent to $x$. We have $G_{e} \subset G_{\varepsilon} \subset G_{X}$. If $G_{X}$ is universally elliptic, so is $G_{\varepsilon}$. Otherwise, $G_{x}$ is flexible. It is associated to a 2 -orbifold $S$ as described in Section 7, and $G_{e}$ has finite index in a boundary subgroup $B$. Since $B$ is the unique maximal small subgroup of $G_{x}$ containing $G_{e}$, it also contains $G_{\varepsilon}$. Thus $G_{e}$ has finite index in $G_{\varepsilon}$, and $G_{\varepsilon}$ is universally elliptic because $G_{e}$ is.

Given any $\mathcal{E}$-tree $T$, we now construct a common refinement $\hat{T}$ of $T_{c}$ and $T$ by blowing up $T_{c}$ as in the proof of Proposition 8.1. There are several steps.

Step 1 We first define a $G_{p}$-invariant subtree $Z_{p} \subset T$, for $p$ a vertex of $T_{c}$.

If $p \in V_{0}\left(T_{c}\right)$, the group $G_{p}$ is not in $\mathcal{E}$. Consider its action on $T$. It fixes a unique point, or it is a flexible vertex group of $T_{J}$ and has a minimal invariant subtree in $T$ (because it is finitely generated). We define $Z_{p}$ as that point or subtree.

If $p \in V_{1}\left(T_{c}\right)$, then $G_{p}$ belongs to $\mathcal{E}$. The cylinder of $T_{J}$ defining $p$ corresponds to an equivalence class $\mathcal{C} \in \mathcal{E} / \sim$ (which contains $G_{p}$ ) as in Section 4.2. If this class corresponds to a cylinder of $T$ (ie if there is an edge of $T$ with stabilizer equivalent to $G_{p}$ ), we define $Z_{p}$ as that cylinder. If not, we now show that $G_{p}$ fixes a point of $T$; this point is necessarily unique (otherwise, there would be a cylinder), and we take it as $Z_{p}$.

Recall that $G_{p}$ is in $\mathcal{E}$, hence is abelian or slender. If it does not fix a point in $T$, it fixes an end or preserves a line. Furthermore, it contains a subgroup $G_{e}$, for $e$ an edge of $T_{J}$. This subgroup is elliptic in $T$ because $T_{J}$ is universally elliptic. Being contained in $G_{p}$, the group $G_{e}$ fixes an end or preserves a line in $T$, so some subgroup of index at most 2 of $G_{e}$ fixes an edge of $T$. The stabilizer of this edge is equivalent to $G_{e}$, hence to $G_{p}$. It yields a cylinder associated to $\mathcal{C}$, a contradiction. 
Step 2 We now explain how to attach edges of $T_{c}$ to $T_{1}=\bigsqcup_{p \in V\left(T_{c}\right)} Z_{p}$. Let $\varepsilon=p q$ be an edge, with $p \in V_{0}\left(T_{c}\right)$ and $q \in V_{1}\left(T_{c}\right)$. We show that $G_{\varepsilon}$ fixes a unique point $x_{\varepsilon}$ in $Z_{p}$, and this point $x_{\varepsilon}$ belongs to $Z_{q}$; we then attach the endpoints of $\varepsilon$ to the copies of $x_{\varepsilon}$ in $Z_{p}$ and $Z_{q}$.

Note that $G_{\varepsilon}$ is elliptic in $T$ (because $T_{c}$ is universally elliptic), and preserves $Z_{p}$ and $Z_{q}$. If $Z_{p}$ is not a point, then $G_{p}$ is flexible, so is an extension $F \rightarrow G_{p} \rightarrow \Sigma$. As explained above, $G_{\varepsilon}$ is contained in a boundary subgroup $B_{0} \subset G_{p}$ with finite index.

We consider the action of $G_{p}$ on its minimal subtree $Z_{p} \subset T$. Every boundary subgroup $B \subset G_{p}$ contains some $G_{e}$ with finite index (with $e$ an edge of $T_{J}$ ), hence acts elliptically. Being normal and finite, the group $F$ acts as the identity, so there is an induced action of $\Sigma$ on $Z_{p}$. For that action, boundary subgroups of $\Sigma$ are elliptic, and edge stabilizers are finite or two-ended because they are slender (resp. abelian). This implies that $B_{0}$, hence also $G_{\varepsilon}$, fixes a unique point $x_{\varepsilon}$ of $Z_{p}$ (see [21, Theorem III.2.6] for the case of surface groups; the extension to an orbifold group is straightforward, as it contains a surface group with finite index).

We now show $x_{\varepsilon} \in Z_{q}$. If not, $G_{\varepsilon}$ fixes the initial edge $e$ of the segment joining $x_{\varepsilon}$ to its projection onto $Z_{q}$. The stabilizer of $e$ is equivalent to $G_{q}$ because $G_{e} \sim G_{\varepsilon} \sim G_{q}$, and $Z_{q}$ was defined as the cylinder containing $e$, so it contains $x_{\varepsilon}$.

Step 3 We can now construct $\widehat{T}$ by gluing edges of $T_{c}$ to $T_{1}$ as in the proof of Proposition 8.1. It refines $T_{c}$, and there is a natural map $g: \hat{T} \rightarrow T$ which is constant on all the edges corresponding to the edges of $T_{c}$, and which is isometric in restriction to each $Z_{p}$. To show that it is a collapse map, it suffices to see that $Z_{p}$ and $Z_{p^{\prime}}$ (viewed as subtrees of $T$ ) cannot have an edge $e$ in common if $p, p^{\prime}$ are distinct vertices of $T_{c}$.

We assume they do, and we reach a contradiction. Let $e_{p} \subset \widehat{T}$ be the copy of $e$ in $Z_{p}$. Then $G_{e_{p}} \subset G_{e}$. One has $G_{e_{p}} \in \mathcal{E}$ because $\mathcal{E}$ is substable, and Axiom (2) implies $G_{e} \sim G_{e_{p}}$. Similarly, $G_{e} \sim G_{e_{p}^{\prime}}$. This also shows that $\widehat{T}$ is an $\mathcal{E}$-tree.

Note that $p$ and $p^{\prime}$ cannot both belong to $V_{1}\left(T_{c}\right)$, as $Z_{p}$ and $Z_{p^{\prime}}$ then are points or distinct cylinders of $T$. We may therefore assume $p \in V_{0}\left(T_{c}\right)$. Let $\varepsilon$ be the initial edge of the segment $\left[p, p^{\prime}\right] \subset T_{c}$. By connectedness of cylinders, the segment joining $e_{p}$ to $e_{p^{\prime}}$ is contained in a cylinder of $\widehat{T}$. Since this segment contains the edge of $\widehat{T}$ corresponding to $\varepsilon$, we have $G_{\varepsilon} \sim G_{e_{p}}$. Thus $\left\langle G_{\varepsilon}, G_{e_{p}}\right\rangle$ is a small subgroup of $G_{p}$, and therefore is contained with finite index in a boundary subgroup $B$. By [21], $G_{e_{p}}$ fixes a unique point of $Z_{p}$, contradicting the fact that $G_{e_{p}}$ fixes $e_{p}$. 


\section{References}

[1] B H Bowditch, Cut points and canonical splittings of hyperbolic groups, Acta Math. 180 (1998) 145-186 MR1638764

[2] I Bumagin, O Kharlampovich, A Miasnikov, The isomorphism problem for finitely generated fully residually free groups, J. Pure Appl. Algebra 208 (2007) 961-977 MR2283438

[3] M Carette, The automorphism group of accessible groups, to appear in J. London Math. Soc. arXiv:0810.0043v1

[4] C Champetier, L'espace des groupes de type fini, Topology 39 (2000) 657-680 MR1760424

[5] M Clay, Contractibility of deformation spaces of $G$-trees, Algebr. Geom. Topol. 5 (2005) 1481-1503 MR2186106

[6] M Clay, M Forester, Whitehead moves for G-trees, Bull. Lond. Math. Soc. 41 (2009) 205-212 MR2496498

[7] M Culler, K Vogtmann, Moduli of graphs and automorphisms of free groups, Invent. Math. 84 (1986) 91-119 MR830040

[8] M J Dunwoody, M E Sageev, JSJ-splittings for finitely presented groups over slender groups, Invent. Math. 135 (1999) 25-44 MR1664694

[9] M Forester, Deformation and rigidity of simplicial group actions on trees, Geom. Topol. 6 (2002) 219-267 MR1914569

[10] M Forester, On uniqueness of JSJ decompositions of finitely generated groups, Comment. Math. Helv. 78 (2003) 740-751 MR2016693

[11] K Fujiwara, P Papasoglu, JSJ-decompositions of finitely presented groups and complexes of groups, Geom. Funct. Anal. 16 (2006) 70-125 MR2221253

[12] V Guirardel, Reading small actions of a one-ended hyperbolic group on $\mathbf{R}$-trees from its JSJ splitting, Amer. J. Math. 122 (2000) 667-688 MR1771568

[13] V Guirardel, Limit groups and groups acting freely on $\mathbb{R}^{n}$-trees, Geom. Topol. 8 (2004) 1427-1470 MR2119301

[14] V Guirardel, G Levitt, Deformation spaces of trees, Groups Geom. Dyn. 1 (2007) 135-181 MR2319455

[15] V Guirardel, G Levitt, A general construction of JSJ decompositions, from: "Geometric group theory", (G Arzhantseva, L Bartholdi, J Burillo, E Ventura, editors), Trends Math., Birkhäuser, Basel (2007) 65-73 MR2395790

[16] V Guirardel, G Levitt, Scott and Swarup's regular neighborhood as a tree of cylinders, Pacific J. Math. 245 (2010) 79-98 MR2602683

[17] V Guirardel, G Levitt, JSJ decompositions: definitions, existence and uniqueness. I: The JSJ deformation space arXiv:0911.3173v2 
[18] V Guirardel, G Levitt, JSJ decompositions: definitions, existence and uniqueness. II: Compatibility and acylindricity arXiv:1002.4564

[19] V Guirardel, G Levitt, Splittings and automorphisms of relatively hyperbolic groups, in preparation

[20] G Levitt, Automorphisms of hyperbolic groups and graphs of groups, Geom. Dedicata 114 (2005) 49-70 MR2174093

[21] J W Morgan, P B Shalen, Valuations, trees, and degenerations of hyperbolic structures. I, Ann. of Math. (2) 120 (1984) 401-476 MR769158

[22] D V Osin, Elementary subgroups of relatively hyperbolic groups and bounded generation, Internat. J. Algebra Comput. 16 (2006) 99-118 MR2217644

[23] P Papasoglu, E Swenson, Boundaries and JSJ decompositions of CAT(0)-groups, Geom. Funct. Anal. 19 (2009) 559-590 MR2545250

[24] F Paulin, Sur la théorie élémentaire des groupes libres (d'après Sela), Astérisque 294, Soc. Math. France (2004) MR2111650

[25] E Rips, Z Sela, Cyclic splittings of finitely presented groups and the canonical JSJ decomposition, Ann. of Math. (2) 146 (1997) 53-109 MR1469317

[26] P Scott, G A Swarup, Regular neighbourhoods and canonical decompositions for groups, Astérisque 289, Soc. Math. France (2003) MR2032389

[27] Z Sela, Acylindrical accessibility for groups, Invent. Math. 129 (1997) 527-565 MR1465334

[28] Z Sela, Structure and rigidity in (Gromov) hyperbolic groups and discrete groups in rank 1 Lie groups. II, Geom. Funct. Anal. 7 (1997) 561-593 MR1466338

[29] Z Sela, Diophantine geometry over groups. VII. The elementary theory of a hyperbolic group, Proc. Lond. Math. Soc. (3) 99 (2009) 217-273 MR2520356

VG: Institut de Mathématiques de Toulouse, Université de Toulouse CNRS (UMR 5219) 118 route de Narbonne, F-31062 Toulouse cedex 9, France

Institut de Recherche Mathématiques de Rennes, Université de Rennes 1 CNRS (UMR 6625) 263 avenue du General Leclerc, CS 74205, 35042 Rennes Cedex, France

GL: Laboratoire de Mathématiques Nicolas Oresme, Université de Caen CNRS (UMR 6139) BP 5186, F-14032 Caen Cedex, France

vincent.guirardel@univ-rennes1.fr, levitt@unicaen.fr

http://perso.univ-rennes1.fr/vincent.guirardel/

Proposed: Martin Bridson

Seconded: Benson Farb, Danny Calegari
Received: 10 December 2008 Accepted: 29 March 2011 